\title{
On the Gribov Problem for Generalized Connections
}

\author{
Christian Fleischhack* \\ Institut für Theoretische Physik \\ Universität Leipzig \\ Augustusplatz 10/11 \\ 04109 Leipzig, Germany \\ Max-Planck-Institut für Mathematik in den Naturwissenschaften \\ Inselstraße 22-26 \\ 04103 Leipzig, Germany
}

October 10, 2001

\begin{abstract}
The bundle structure of the space $\overline{\mathcal{A}}$ of Ashtekar's generalized connections is investigated in the compact case. It is proven that every stratum is a locally trivial fibre bundle. The only stratum being a principal fibre bundle is the generic stratum. Its structure group equals the space $\overline{\mathcal{G}}$ of all generalized gauge transforms modulo the constant center-valued gauge transforms. For abelian gauge theories the generic stratum is globally trivial and equals the total space $\overline{\mathcal{A}}$. However, for a certain class of non-abelian gauge theories - e.g., all $S U(N)$ theories - the generic stratum is nontrivial. This means, there are no global gauge fixings - the so-called Gribov problem. Nevertheless, there is a covering of the generic stratum by trivializations each having total induced Haar measure 1 .
\end{abstract}

\section{Keywords}

Gribov Problem, Ashtekar Connections, Generic Connections, Gauge Fixing, Ashtekar-Lewandowski Measure

PASC

11.15.Tk, 02.30. $\mathrm{Cj}, 02.40 .-\mathrm{k}$

MSC 2000

$81 \mathrm{~T} 13$ (Primary) 53C05, 55R10, 57R22, 58D19 (Secondary)

*e-mail: fleisch@itp.uni-leipzig.de or chfl@mis.mpg.de 


\section{Introduction}

The functional integral approach to quantum field theories consists of two basic steps: first the determination of a "physical" (Euclidian) measure on the configuration space and second the reconstruction of the quantum theory via an Osterwalder-Schrader procedure. In this paper we will focus on a certain issue arising in the first step - the Gribov problem.

The configuration space of smooth gauge field theories has a very complicated structure. The space $\mathcal{A} / \mathcal{G}$ of all smooth gauge orbits is, in general, neither affine, nor compact, nor a manifold. In contrast, the space $\mathcal{A}$ of all smooth gauge fields (mathematically, connections) is an affine space, and therefore (from the mathematical point of view) by far more pleasant. That is why in the 60s Faddeev and Popov [11 proposed the following strategy for the gauge-invariant formulation of functional integrals: First one defines on $\mathcal{A}$ the formal measure $\mathcal{D} A=\prod_{x, \mu} \mathrm{d} A^{\mu}(x)$. Due to the gauge invariance of the YangMills action $S_{\mathrm{YM}}$ the functional integral $\int \mathrm{e}^{\mathrm{i} S_{\mathrm{YM}}(A)} \mathcal{D} A$ has to be naively proportional to the integral $\int \mathcal{D} g \equiv \int \prod_{x} \mathrm{~d} g(x)$ over all gauge transforms. In order to separate this unphysical factor, one chooses a gauge fixing, e.g. (as Faddeev and Popov did) $\partial_{\nu} A^{\nu}=0$, and gets $\prod_{x, \mu} \mathrm{d} A_{\mu}(x)=\Delta(A) \prod_{x}\left(\delta\left(\partial_{\nu} A^{\nu}(x)\right) \prod_{\mu} \mathrm{d} A^{\mu}(x)\right) \prod_{x} \mathrm{~d} g(x)$. The so called FaddeevPopov determinant $\Delta(A)$, a kind of a degenerate Jacobi determinant, has now to be chosen such that $\Delta(A) \prod_{x} \delta\left(\partial_{\nu}(A \circ g)^{\nu}(x)\right) \mathrm{d} g(x)$ becomes a constant independent of $A$. Finally, $\prod_{x}\left(\delta\left(\partial_{\nu} A^{\nu}(x)\right) \prod_{\mu} \mathrm{d} A^{\mu}(x)\right)$ is to be considered as an equivalent of a measure on $\mathcal{A} / \mathcal{G}$.

About ten years later, Gribov [15] noticed a severe problem: The Coulomb gauge is (for certain gauge theories) no gauge fixing since in some gauge orbits several connections fulfill the gauge condition. Therefore, besides the Faddeev-Popov determinant a corresponding, highly nontrivial factor had to be inserted into the functional integral. Now, one was hoping to avoid this problem using another gauge fixing. But this hope broke down a few months later: Singer [27 investigated this problem more systematically and found that the nonexistence of gauge fixings is a typical property of gauge theories with non-compact structure group. Mathematically, this means simply that there is no global section in the foliation $\mathcal{A} \longrightarrow \mathcal{A} / \mathcal{G}$ of the set of connections over the gauge orbit space. Moreover, Singer was able to prove that (for structure group $\mathbf{G}=S U(N)$ and spacetime manifold $M=S^{4}$ ) there is no global section even in the principal fibre bundle of all irreducible connections.

Most of these problems can be formulated and could arise in a similar way also in the Ashtekar framework which differs (in the gauge-theoretical sense) from the standard one mainly in the usage of distributional connections instead of only smooth ones. Therefore we want to study in the present paper whether there is a Gribov problem for Ashtekar connections as well and, if necessary, study its impact. Our considerations can be understood as a continuation of the investigations started in [13, 12] on the structure of the configuration space of generalized gauge theories.

\subsection{Preliminaries}

Before we outline the paper we recall the basic facts and notations from [13] where the details can be found. Other references are [1, 2, 3].

Let the "space-time" $M$ be an at least two-dimensional manifold, $m \in M$ some fixed point and the structure group $\mathbf{G}$ be a compact and (for technical reasons) connected Lie group. $\mathcal{P}$ 
denotes the groupoid of all paths in $M, \mathcal{H G}$ the group of all paths starting and ending in $m$. The set $\overline{\mathcal{A}}$ of generalized connections $\bar{A}$ is defined by $\overline{\mathcal{A}}:=\lim _{\Gamma} \mathbf{G} \# \mathbf{E}(\Gamma)=\operatorname{Hom}(\mathcal{P}, \mathbf{G})$. Here $\Gamma$ runs over all finite graphs in $M . \mathbf{E}(\Gamma)$ is the set of edges in $\Gamma, \mathbf{V}(\Gamma)$ will be that of all vertices. The set $\overline{\mathcal{G}}$ of generalized gauge transforms $\bar{g}$ is $\overline{\mathcal{G}}:=\lim _{\Gamma} \mathbf{G}^{\# \mathbf{V}(\Gamma)}=\operatorname{Maps}(M, \mathbf{G})$. It acts continuously on $\overline{\mathcal{A}}$ via $h_{\bar{A} \circ \bar{g}}(\gamma)=g_{\gamma(0)}^{-1} h_{\bar{A}}(\gamma) g_{\gamma(1)}$ where the path $\gamma$ is in $\mathcal{P}$ and $h_{\bar{A}}$ is the homomorphism corresponding to $\bar{A}$. The stabilizer $\mathbf{B}(\bar{A})$ of $\bar{A}$ contains exactly those gauge transforms that fulfill $h_{\bar{A}}\left(\gamma_{x}\right)=g_{m}^{-1} h_{\bar{A}}\left(\gamma_{x}\right) g_{x}$ for all $x \in M$ and whose $m$-component $g_{m}$ lies in the holonomy centralizer $Z\left(\mathbf{H}_{\bar{A}}\right)$ of $\bar{A}$, i.e. the centralizer of the holonomy group of $\bar{A}$. Here, for all $x, \gamma_{x}$ is some fixed path from $m$ to $x$. We have $\mathbf{B}(\bar{A}) \cong Z\left(\mathbf{H}_{\bar{A}}\right)$. Now, the orbit type of $\bar{A}$ is defined to be the $\overline{\mathcal{G}}$-conjugacy class of $\mathbf{B}(\bar{A})$, but equivalently it can be defined to be the $\mathbf{G}$-conjugacy class $\left[Z\left(\mathbf{H}_{\bar{A}}\right)\right]$ of the holonomy centralizer of $\bar{A}$. This definition will be used in the following. The types are partially ordered by the natural inclusion-induced ordering of classes of subgroups of $\mathbf{G}$. A stratum $\overline{\mathcal{A}}_{=t}$ is the set of all connections $\bar{A} \in \overline{\mathcal{A}}$ having type $t$ and the generic stratum $\overline{\mathcal{A}}_{\text {gen }}$ is the set of all connections having the maximal orbit type $[Z]$ where $Z \equiv Z(\mathbf{G})$ is the center of $\mathbf{G} . \overline{\mathcal{A}}_{\text {gen }}$ is an open, dense and $\overline{\mathcal{G}}$-invariant subset of $\overline{\mathcal{A}}$. Furthermore, there is a slice theorem on $\overline{\mathcal{A}}$. This means, for every $\bar{A} \in \overline{\mathcal{A}}$ there is a so-called slice $\bar{S} \subseteq \overline{\mathcal{A}}$ with $\bar{A} \in \bar{S}$ such that:

- $\bar{S} \circ \overline{\mathcal{G}}$ is an open neighbourhood of $\bar{A} \circ \overline{\mathcal{G}}$ and

- there is an equivariant retraction $F: \bar{S} \circ \overline{\mathcal{G}} \longrightarrow \bar{A} \circ \overline{\mathcal{G}}$ with $F^{-1}(\{\bar{A}\})=\bar{S}$.

The most important tool for the proof of this theorem has been a so-called reduction mapping $\varphi_{\boldsymbol{\alpha}}$. Note, due to the compactness of $\mathbf{G}$, any centralizer is finitely generated and consequently there is a finite set $\boldsymbol{\alpha} \subseteq \mathcal{H G}$ of paths starting and ending in $m$ such that $Z\left(h_{\bar{A}}(\boldsymbol{\alpha})\right)=Z\left(\mathbf{H}_{\bar{A}}\right)$. Since $Z\left(h_{\bar{A}}(\boldsymbol{\alpha})\right)$ is the orbit type of $h_{\bar{A}}(\boldsymbol{\alpha})$ w.r.t. the adjoint action of $\mathbf{G}$ on $\mathbf{G}^{\# \boldsymbol{\alpha}}$, the reduction

mapping $\varphi_{\boldsymbol{\alpha}}: \overline{\mathcal{A}} \longrightarrow \mathbf{G}^{\# \boldsymbol{\alpha}}$ with $\bar{A}^{\prime} \longmapsto h_{\bar{A}^{\prime}}(\boldsymbol{\alpha})$ lifts the slice theorem from $\mathbf{G}^{\# \boldsymbol{\alpha}}$ to $\overline{\mathcal{A}}$. The notion of a reduction mapping will be crucial again in the present paper.

Finally we note that there is a natural measure $\mu_{0}$ on $\overline{\mathcal{A}}$, the so-called Ashtekar-Lewandowski measure or induced Haar measure [12, 2]. At the moment, we should only notice that $\mu_{0}\left(\pi_{\Gamma}^{-1}(U)\right)=\mu_{\text {Haar }}(U)$ for all graphs $\Gamma$ with corresponding projections $\pi_{\Gamma}: \overline{\mathcal{A}} \longrightarrow \mathbf{G}^{\# \mathbf{E}(\Gamma)}$, $\pi_{\Gamma}(\bar{A})=\left(h_{\bar{A}}\left(e_{1}\right), \ldots, h_{\bar{A}}\left(e_{\# \mathbf{E}(\Gamma)}\right)\right)$, and all measurable $U \subseteq \mathbf{G}^{\# \mathbf{E}(\Gamma)}$.

\subsection{Outline of the Paper}

The guideline for our paper will be given by the following five questions.

Question 1 Is $\pi: \overline{\mathcal{A}} \longrightarrow \overline{\mathcal{A}} / \overline{\mathcal{G}}$ a fibre bundle?

In general, the answer is negative; at least, if we demand, that all fibres $\bar{A} \circ \overline{\mathcal{G}}$ have to be isomorphic $\overline{\mathcal{G}}$-spaces. This required [6] that all stabilizers are $\overline{\mathcal{G}}$-conjugate. But, this implies 13. that all Howe subgroups of $\mathbf{G}$ have to be $\mathbf{G}$-conjugate. This, however, is only possible for abelian $\mathbf{G}$.

For arbitrary $\mathbf{G}$ we can expect bundle structures only on subsets of $\overline{\mathcal{A}}$ where all connections have conjugate stabilizers. The maximal sets of that kind are exactly the sets of connections having one and the same gauge orbit type $t$, i.e. the strata $\overline{\mathcal{A}}_{=t}$.

Question 2 Are the strata $\pi: \overline{\mathcal{A}}_{=t} \longrightarrow \overline{\mathcal{A}}_{=t} / \overline{\mathcal{G}}$ locally trivial fibre bundles?

It is well-known that for actions of compact Lie groups on arbitrary (completely regular) spaces the strata are always fibre bundles. However, the proof of this theorem does not 
require the Lie property of the acting group, but the existence of a slice theorem. In contrast to the former one the latter one is guaranteed also in our case of interest [13]. Hence, we will be able to prove that the strata are indeed fibre bundles.

The next stronger question is

Question 3 What strata are principal fibre bundles?

For a fibre bundle to be a principal fibre bundle, at least the structure group of that bundle has to equal the typical fibre. Since a stratum $\overline{\mathcal{A}}_{=t}$ has (for general reasons) the typical fibre $\mathbf{B}(\bar{A}) \backslash \overline{\mathcal{G}}$ and structure group $\mathbf{B}(\bar{A}) \backslash N\left(\mathbf{B}(\bar{A})\right.$ ) (for some $\bar{A} \in \overline{\mathcal{A}}_{=t}$ ), the stabilizer $\mathbf{B}(\bar{A})$ has to be a normal subgroup in $\overline{\mathcal{G}}$. (Here, $N(\mathbf{B}(\bar{A})$ ) denotes the normalizer of $\mathbf{B}(\bar{A})$ w.r.t. $\overline{\mathcal{G}}$.) We will show that this is the case in the generic stratum only.

As every other stratum, the generic stratum is locally trivial.

Question 4 Is $\pi: \overline{\mathcal{A}}_{\text {gen }} \longrightarrow \overline{\mathcal{A}}_{\text {gen }} / \overline{\mathcal{G}}$ even globally trivial?

For smooth connections - as mentioned above - this is not the case; this is just the Gribov problem.

In the case of generalized connections the reduction mapping will play again the most important rôle. By its means we can reduce the problem of the triviality of the generic stratum in $\overline{\mathcal{A}}$ to the corresponding problem for the generic stratum in $\mathbf{G}^{k}$, i.e. the set of all $\vec{g} \in \mathbf{G}^{k}$ with $Z(\vec{g})=Z(\mathbf{G})$. Namely, by the finiteness lemma for centralizers [13 we find finitely many $g_{i} \in \mathbf{G}$ with $Z(\vec{g}) \equiv Z\left(\left\{g_{1}, \ldots, g_{k}\right\}\right)=Z(\mathbf{G})$. Now we choose a graph $\Gamma$ having $k$ edges $\alpha_{i} \in \mathcal{H G}$ and one vertex $m$ and denote the corresponding reduction mapping $\pi_{\Gamma}: \overline{\mathcal{A}} \longrightarrow \mathbf{G}^{k}$ simply by $\varphi$. Since $\varphi$ is surjective even on $\overline{\mathcal{A}}_{\text {gen }}$ [13], we get the following commutative diagram

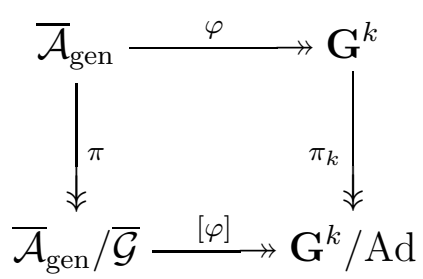

One could now conjecture that the generic stratum should be nontrivial because otherwise one would get a trivialization of $\pi_{k}: \mathrm{G}^{k} \longrightarrow \mathrm{G}^{k} / \mathrm{Ad}$, although in general $\pi_{k}$ is not a fibre bundle. However, this argumentation is incorrect; namely, there is no "gauge invariant" section for $\varphi$, i.e. no induced section for $[\varphi]$. But, restricting $\mathbf{G}^{k}$ in the diagram above to the generic elements (and modify the remaining three spaces accordingly to the given maps) this obstruction disappears. Indeed, we will be able to prove that $\pi: \overline{\mathcal{A}}_{\text {gen }} \longrightarrow \overline{\mathcal{A}}_{\text {gen }} / \overline{\mathcal{G}}$ is nontrivial, as far as $\pi_{k}:\left(\mathbf{G}^{k}\right)_{\text {gen }} \longrightarrow\left(\mathbf{G}^{k}\right)_{\text {gen }} /$ Ad is nontrivial. Moreover, we will explicitely determine a couple of groups (among them $\mathbf{G}=S U(N)$ ) that fulfill the criterion. Hence we will prove that the Gribov problem appears for generalized connections again.

In the case of continuous connections the impact of the Gribov problem to the calculation of functional integrals is enormous. Since for the integration trivial subbundles are of special interest, we have the next natural

Question 5 What "size" can trivial subbundles of $\overline{\mathcal{A}}_{\text {gen }}$ have at most? 
Using the reduction mapping above we can show that the triviality of $\pi_{k}$ over $V \subseteq\left(\mathbf{G}^{k}\right)_{\text {gen }}$ implies the triviality of $\pi$ over $\varphi^{-1}(V) \subseteq \overline{\mathcal{A}}_{\text {gen }}$. Consequently we get as a by-product that $\pi$ is trivial on the whole $\overline{\mathcal{A}}_{\text {gen }} \equiv \overline{\mathcal{A}}$ for abelian $\mathbf{G}$. In the general case our task will be the search for domains where $\pi_{k}$ is trivial. The idea for that comes from the fact that a smooth principal fibre bundle over a contractible manifold is always trivial. Hence we triangulate $\left(\mathbf{G}^{k}\right)_{\operatorname{gen}} / \mathrm{Ad}$, cut out the lower-dimensional simplices (this is in particular a set of [Lebesgue] measure 0) and get a disjoint union $W$ of contractible manifolds. The preimage of that union by $\pi_{k} \circ \varphi$ yields the desired trivialization $U \subseteq \overline{\mathcal{A}}_{\text {gen }}$ with $\mu_{0}(U)=\mu_{\text {Haar }}\left(\pi_{k}^{-1}(W)\right)=1$.

This way we have found a trivial subbundle arosen from $\overline{\mathcal{A}}$ by cutting out certain zero subsets. This means that for generalized connections the Gribov problem is completely irrelevant for the calculation of functional integrals supposed the considered measure on $\overline{\mathcal{A}}$ is absolutely continuous w.r.t. the induced Haar measure.

Up to this limitation we get for generalized connections:

The Gribow problem appears, but it causes no longer a problem.

In this paper we investigate first the fibre bundle structure of the strata and prove that the generic stratum is the only stratum having the structure of a principal fibre bundle. Next, we show that $\mathbf{G}^{k}$ for sufficiently large $k$ is globally trivial up to a zero subset and transfer this statement to $\overline{\mathcal{A}}$. The proof is quite technical, although its idea sketched above is rather simple. Then we prove the triviality of $\overline{\mathcal{A}}$ in the abelian case. Finally, we state a grouptheoretical criterion for the nontriviality of $\overline{\mathcal{A}}_{\text {gen }}$ in the nonabelian case and discuss which concrete $\mathbf{G}$ fulfill this criterion. The example $\mathbf{G}=S U(2)$ will be described in Appendix $\mathbf{B}$ in detail.

\section{Fibre Bundle Structure of the Strata}

Proposition 2.1 Let $t \in \mathcal{T}$ be a gauge orbit type and $\bar{A} \in \overline{\mathcal{A}}_{=t}$ be some connection of that type.

Then the stratum $\overline{\mathcal{A}}_{=t}$ is a locally trivial fibre bundle over $\overline{\mathcal{A}}_{=t} / \overline{\mathcal{G}}$ with fibre $\mathbf{B}(\bar{A}) \backslash \overline{\mathcal{G}}$ and structure group $\mathbf{B}(\bar{A}) \backslash N(\mathbf{B}(\bar{A}))$ acting on the typical fibre by left translation.

Proof We know from [13] that there is a slice theorem on $\overline{\mathcal{A}}$. Now the assertion follows immediately because in general every stratum of a space with slice theorem is such a fibre bundle (cf. Appendix D). $\quad$ qed

Remark The preceding proposition is a further generalization of a result for regular connections to the Ashtekar approach. Already in 1978, Daniel and Viallet [8] noticed that classically gauge fixings always exist at least locally. This result was confirmed by the later statements [25, 23, 20, 21] on the triviality of the strata.

We know already from [13] that a lot of structures in $\overline{\mathcal{G}}$ can be reduced to structures in the Lie group $\mathbf{G}$. For instance, we have seen that $\mathbf{B}(\bar{A})$ is always isomorphic to $Z\left(\mathbf{H}_{\bar{A}}\right)$. This, the other way round, implies that the homeomorphy class of each orbit is determined not only by $\mathbf{B}(\bar{A}) \backslash \overline{\mathcal{G}}$, but also by $Z\left(\mathbf{H}_{\bar{A}}\right) \backslash \mathbf{G}$. We will see now that there is an analogous result for the structure group $\mathbf{B}(\bar{A}) \backslash N(\mathbf{B}(\bar{A}))$. We start investigating $N(\mathbf{B}(\bar{A}))$ itself. 
Proposition 2.2 Let $\bar{A} \in \overline{\mathcal{A}}$ and $\bar{g} \in \overline{\mathcal{G}}$. Furthermore, we again fix for every $x \in M$ a path $\gamma_{x}$ from $m$ to $x$, where $\gamma_{m}$ is trivial.

Then we have $\bar{g} \in N(\mathbf{B}(\bar{A}))$ iff

1. $g_{m} \in N\left(Z\left(\mathbf{H}_{\bar{A}}\right)\right)$ and

2. $g_{x} \in h_{\bar{A}}\left(\gamma_{x}\right)^{-1} Z\left(Z\left(\mathbf{H}_{\bar{A}}\right)\right) g_{m} h_{\bar{A}}\left(\gamma_{x}\right)$ for all $x \in M$.

Proof In general, $\bar{g} \in N(\mathbf{B}(\bar{A})) \Longleftrightarrow \bar{g}^{-1} \mathbf{B}(\bar{A}) \bar{g} \subseteq \mathbf{B}(\bar{A})$.

So let $\bar{g} \in \overline{\mathcal{G}}$ and $\bar{g}^{\prime} \in \mathbf{B}(\bar{A})$. Then we have

$$
\begin{aligned}
& \bar{g}^{-1} \bar{g}^{\prime} \bar{g} \in \mathbf{B}(\bar{A}) \\
& \Longleftrightarrow \quad 1 . g_{m}^{-1} g_{m}^{\prime} g_{m} \in Z\left(\mathbf{H}_{\bar{A}}\right) \\
& \text { 2. } h_{\bar{A}}\left(\gamma_{x}\right)=\left(g_{m}^{-1} g_{m}^{\prime} g_{m}\right)^{-1} h_{\bar{A}}\left(\gamma_{x}\right)\left(g_{x}^{-1} g_{x}^{\prime} g_{x}\right) \quad \forall x \in M \\
& \Longleftrightarrow \text { 1. } g_{m}^{-1} g_{m}^{\prime} g_{m} \in Z\left(\mathbf{H}_{\bar{A}}\right) \\
& \text { 2. } g_{m} h_{\bar{A}}\left(\gamma_{x}\right) g_{x}^{-1} h_{\bar{A}}\left(\gamma_{x}\right)^{-1}=\left(g_{m}^{\prime}\right)^{-1} g_{m} h_{\bar{A}}\left(\gamma_{x}\right) g_{x}^{-1} h_{\bar{A}}\left(\gamma_{x}\right)^{-1} g_{m}^{\prime} \quad \forall x \in M \text {. } \\
& \text { (since } g_{x}^{\prime}=h_{\bar{A}}\left(\gamma_{x}\right)^{-1} g_{m}^{\prime} h_{\bar{A}}\left(\gamma_{x}\right) \text { ) }
\end{aligned}
$$

Hence,

$$
\begin{array}{lll} 
& \bar{g} \in N(\mathbf{B}(\bar{A})) \\
\Longleftrightarrow & \bar{g}^{-1} \bar{g}^{\prime} \bar{g} \in \mathbf{B}(\bar{A}) \quad \forall \bar{g}^{\prime} \in \mathbf{B}(\bar{A}) & \\
& 1 . g_{m} \in N\left(Z\left(\mathbf{H}_{\bar{A}}\right)\right) & \\
\text { 2. } g_{m} h_{\bar{A}}\left(\gamma_{x}\right) g_{x}^{-1} h_{\bar{A}}\left(\gamma_{x}\right)^{-1} \in Z\left(Z\left(\mathbf{H}_{\bar{A}}\right)\right) & \forall x \in M \\
\text { 1. } g_{m} \in N\left(Z\left(\mathbf{H}_{\bar{A}}\right)\right) & \\
2 . \quad g_{x} \in h_{\bar{A}}\left(\gamma_{x}\right)^{-1} Z\left(Z\left(\mathbf{H}_{\bar{A}}\right)\right) g_{m} h_{\bar{A}}\left(\gamma_{x}\right) & \forall x \in M .
\end{array}
$$

Example 1 In the generic stratum we have $Z\left(\mathbf{H}_{\bar{A}}\right)=Z(\mathbf{G})$, hence $N\left(Z\left(\mathbf{H}_{\bar{A}}\right)\right)=\mathbf{G}$ and $Z\left(Z\left(\mathbf{H}_{\bar{A}}\right)\right)=\mathbf{G}$. Consequently, $N(\mathbf{B}(\bar{A}))=\overline{\mathcal{G}}$.

Example 2 In the minimal stratum we have $Z\left(\mathbf{H}_{\bar{A}}\right)=\mathbf{G}$. Hence $N\left(Z\left(\mathbf{H}_{\bar{A}}\right)\right)=\mathbf{G}$ and $Z\left(Z\left(\mathbf{H}_{\bar{A}}\right)\right)=Z(\mathbf{G})$. By the proposition above we have $\bar{g} \in N(\mathbf{B}(\bar{A}))$ iff there is some $g_{m} \in \mathbf{G}$ such that $g_{x} \in h_{\bar{A}}\left(\gamma_{x}\right)^{-1} Z(\mathbf{G}) g_{m} h_{\bar{A}}\left(\gamma_{x}\right)=$ $Z(\mathbf{G}) h_{\bar{A}}\left(\gamma_{x}\right)^{-1} g_{m} h_{\bar{A}}\left(\gamma_{x}\right)$ for all $x \in M$.

Remark We realize already at this point that (for nonabelian $\mathbf{G}$ ) $\mathbf{B}(\bar{A})$ is not a normal subset in $\overline{\mathcal{G}}$ provided $\bar{A}$ has minimal type. A more general statement can be found in Section 1 .

The preceding proposition shows how the normalizer of $\mathbf{B}(\bar{A})$ is determined by objects in the Lie group $\mathrm{G}$.

Corollary 2.3 For all $\bar{A} \in \overline{\mathcal{A}}$ the spaces $N(\mathbf{B}(\bar{A}))$ and $N\left(Z\left(\mathbf{H}_{\bar{A}}\right)\right) \times \mathbf{X}_{x \neq m} Z\left(Z\left(\mathbf{H}_{\bar{A}}\right)\right)$ are homeomorphic.

Proof We see immediately that the map $\Psi_{1}: \bar{g} \longmapsto\left(g_{m},\left(h_{\bar{A}}\left(\gamma_{x}\right) g_{x} h_{\bar{A}}\left(\gamma_{x}\right)^{-1} g_{m}^{-1}\right)_{x \neq m}\right)$ is a homeomorphism between these two spaces. qed

We emphasize that both subgroups of $\overline{\mathcal{G}}$ are in general not isomorphic as topological groups. At least there is no "reasonable" homomorphism. We will discuss this problem a bit more 
in detail in Appendix A. Roughly speaking, the homomorphy property is destroyed by the above restriction on $g_{x}$ to be a (usually non-commutative) product of $g_{m}$ with elements in $Z\left(Z\left(\mathbf{H}_{\bar{A}}\right)\right)$.

In order to investigate the structure of $\mathbf{B}(\bar{A}) \backslash N(\mathbf{B}(\bar{A})$ ), we recall the form of $\mathbf{B}(\bar{A})$. By [13] $\mathbf{B}(\bar{A})$ and $Z\left(\mathbf{H}_{\bar{A}}\right) \times \mathbf{X}_{x \neq m}\left\{e_{\mathbf{G}}\right\}$ are isomorphic topological groups. Hence, heuristically

$$
\mathbf{B}(\bar{A}) \backslash N(\mathbf{B}(\bar{A})) \text { and } Z\left(\mathbf{H}_{\bar{A}}\right) \backslash N\left(Z\left(\mathbf{H}_{\bar{A}}\right)\right) \times \underset{x \neq m}{\times} Z\left(Z\left(\mathbf{H}_{\bar{A}}\right)\right)
$$

are homeomorphic. The group isomorphy, however, is not to be expected: Since the base centralizer $\mathbf{B}(\bar{A})$ and the holonomy centralizer $Z\left(\mathbf{H}_{\bar{A}}\right)$ are isomorphic groups, it is unlikely that there arise isomorphic groups from originally non-isomorphic groups by factorization. Indeed there are examples (generic connections for $\mathbf{G}=S U(2)$ ) admitting no such "reasonable" group isomorphism. We will discuss this further in Appendix A.

Proposition 2.4 For every $\bar{A} \in \overline{\mathcal{A}}$

$$
\begin{aligned}
{\left[\Psi_{1}\right]: \mathbf{B}(\bar{A}) \backslash N(\mathbf{B}(\bar{A})) } & \longrightarrow Z\left(\mathbf{H}_{\bar{A}}\right) \backslash N\left(Z\left(\mathbf{H}_{\bar{A}}\right)\right) \times \times_{x \neq m} Z\left(Z\left(\mathbf{H}_{\bar{A}}\right)\right) \\
{[\bar{g}]_{\mathbf{B}(\bar{A})} } & \longmapsto\left(\left[g_{m}\right]_{Z\left(\mathbf{H}_{\bar{A}}\right)},\left(h_{\bar{A}}\left(\gamma_{x}\right) g_{x} h_{\bar{A}}\left(\gamma_{x}\right)^{-1} g_{m}^{-1}\right)_{x \neq m}\right)
\end{aligned}
$$

is a homeomorphism.

Proof • $\left[\Psi_{1}\right]$ is well-defined.

Let $\left[\bar{g}_{1}\right]_{\mathbf{B}(\bar{A})}=\left[\bar{g}_{2}\right]_{\mathbf{B}(\bar{A})}$, i.e. $\bar{g}_{1}=\bar{g}^{\prime} \bar{g}_{2}$ with $\bar{g}^{\prime} \in \mathbf{B}(\bar{A})$. Then $g_{m}^{\prime} \in Z\left(\mathbf{H}_{\bar{A}}\right)$, hence $\left[g_{1, m}\right]_{Z\left(\mathbf{H}_{\bar{A}}\right)}=\left[g_{m}^{\prime} g_{2, m}\right]_{Z\left(\mathbf{H}_{\bar{A}}\right)}=\left[g_{2, m}\right]_{Z\left(\mathbf{H}_{\bar{A}}\right)}$. Moreover,

$$
\begin{aligned}
h_{\bar{A}}\left(\gamma_{x}\right) g_{1, x} h_{\bar{A}}\left(\gamma_{x}\right)^{-1} g_{1, m}^{-1} & =h_{\bar{A}}\left(\gamma_{x}\right) g_{x}^{\prime} g_{2, x} h_{\bar{A}}\left(\gamma_{x}\right)^{-1} g_{2, m}^{-1}\left(g_{m}^{\prime}\right)^{-1} \\
& =h_{\bar{A}}\left(\gamma_{x}\right) g_{x}^{\prime} h_{\bar{A}}\left(\gamma_{x}\right)^{-1} h_{\bar{A}}\left(\gamma_{x}\right) g_{2, x} h_{\bar{A}}\left(\gamma_{x}\right)^{-1} g_{2, m}^{-1}\left(g_{m}^{\prime}\right)^{-1} \\
& =g_{m}^{\prime} h_{\bar{A}}\left(\gamma_{x}\right) g_{2, x} h_{\bar{A}}\left(\gamma_{x}\right)^{-1} g_{2, m}^{-1}\left(g_{m}^{\prime}\right)^{-1} \\
& =h_{\bar{A}}\left(\gamma_{x}\right) g_{2, x} h_{\bar{A}}\left(\gamma_{x}\right)^{-1} g_{2, m}^{-1}
\end{aligned}
$$

due to the properties of $\bar{g}^{\prime} \in \mathbf{B}(\bar{A})$ [13] and $\bar{g}_{i} \in N(\mathbf{B}(\bar{A}))$.

- $\left[\Psi_{1}\right]$ is surjective and continuous.

Follows immediately from the commutative diagram

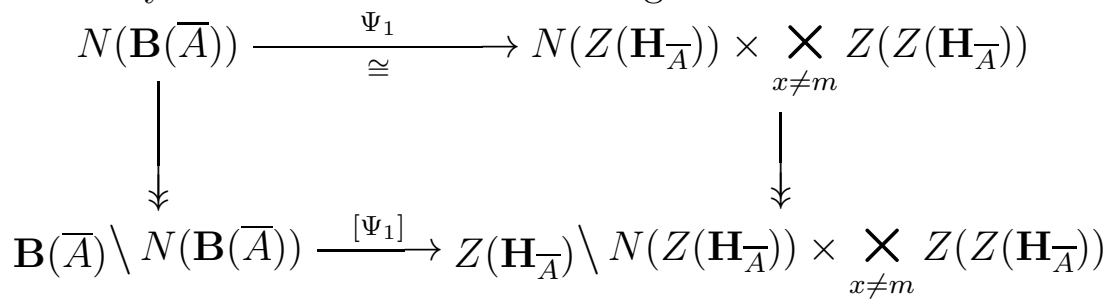

and Corollary 2.3 .

- $\left[\Psi_{1}\right]$ is injective.

Let $\left[\Psi_{1}\right]\left(\left[\bar{g}_{1}\right]\right)=\left[\Psi_{1}\right]\left(\left[\bar{g}_{2}\right]\right)$. Then $g_{1, m} g_{2, m}^{-1} \in Z\left(\mathbf{H}_{\bar{A}}\right)$ and

This implies

$$
h_{\bar{A}}\left(\gamma_{x}\right) g_{2, x} h_{\bar{A}}\left(\gamma_{x}\right)^{-1} g_{2, m}^{-1}=h_{\bar{A}}\left(\gamma_{x}\right) g_{1, x} h_{\bar{A}}\left(\gamma_{x}\right)^{-1} g_{1, m}^{-1} \text {. }
$$

$$
\begin{aligned}
g_{2, m} h_{\bar{A}}\left(\gamma_{x}\right) g_{2, x}^{-1} & =g_{1, m} h_{\bar{A}}\left(\gamma_{x}\right) g_{1, x}^{-1} \\
& =g_{1, m} g_{2, m}^{-1} g_{2, m} h_{\bar{A}}\left(\gamma_{x}\right) g_{2, x}^{-1} h_{\bar{A}}\left(\gamma_{x}\right)^{-1} h_{\bar{A}}\left(\gamma_{x}\right) g_{2, x} g_{1, x}^{-1} \\
& =g_{2, m} h_{\bar{A}}\left(\gamma_{x}\right) g_{2, x}^{-1} h_{\bar{A}}\left(\gamma_{x}\right)^{-1} g_{1, m} g_{2, m}^{-1} h_{\bar{A}}\left(\gamma_{x}\right) g_{2, x} g_{1, x}^{-1}
\end{aligned}
$$

using $\bar{g}_{2} \in N(\mathbf{B}(\bar{A}))$ and $g_{1, m} g_{2, m}^{-1} \in Z\left(\mathbf{H}_{\bar{A}}\right)$. Consequently,

thus $\bar{g}_{1} \bar{g}_{2}^{-1} \in \mathbf{B}(\bar{A})$.

$$
h_{\bar{A}}\left(\gamma_{x}\right)=\left(g_{1, m} g_{2, m}^{-1}\right)^{-1} h_{\bar{A}}\left(\gamma_{x}\right) g_{1, x} g_{2, x}^{-1}
$$


- $\left[\Psi_{1}\right]$ is a homeomorphism.

$N(\mathbf{B}(\bar{A}))$ is a closed subgroup of $\overline{\mathcal{G}}$, hence compact. Thus, $\mathbf{B}(\bar{A}) \backslash N(\mathbf{B}(\bar{A}))$ is compact as well, hence $\left[\Psi_{1}\right]$ is a continuous map of a compact space to a Hausdorff space. This gives [19] the assertion.

qed

\section{Modified Principal Fibre Bundles}

Now we will find out which strata are even principal fibre bundles. Here we will use a slightly modified definition for principal fibre bundles. Actually, one makes the demand on such a bundle that the structure group acts freely on the fibres, i.e. all stabilizers are to be trivial. This will not be the case for generalized connections in general because every holonomy centralizer - being isomorphic to the corresponding stabilizer - contains at least the center of G. Therefore we will factor out exactly these "bugging" parts:

Definition 3.1 A $G$-space $X$ is called principal $G$-fibre bundle iff all $x \in X$ have the same stabilizer $S$.

The structure group of $\pi$ is $S \backslash G$.

Sometimes we say that also $\pi: X \longrightarrow X / G$ is a principal $G$-fibre bundle.

Proposition 3.1 Let (in the notation above) $X$ be a principal fibre bundle with "typical" stabilizer $S$. Then $S$ is a normal subgroup in $G$, i.e., $S \backslash G$ is a topological group.

In a natural manner $S \backslash G$ acts continuously and freely on $X$. Moreover, $X / G \cong X /(S \backslash G)$.

This way the definition of a (not necessarily locally trivial) principal fibre bundle above is equivalent to the usual one. Here neither the total nor the base space gets changed. Only the acting group is reduced to its part being essential for the action. By the proposition above we see that the notation "structure group" is reasonable. It coincides with the standard definition for fibre bundles. Note, however, that the $G$ in a "principal $G$-fibre bundle" $X$ does not denote the structure group as usual, but the total acting group.

Proof Let $x \in X$ and $g \in G$. Obviously, the stabilizer of $x \circ g$ equals $G_{x \circ g}=g^{-1} G_{x} g=$ $g^{-1} S g$. Since $X$ is a principal fibre bundle, we have $g^{-1} S g=S$, i.e., $S$ is a normal subgroup.

Obviously, the action $x \circ[g]_{S}:=x \circ g$ is well-defined and continuous. Since from $x \circ[g]_{S}=x$ we get $x \circ g=x$, hence $g \in S$, the action is free. The homeomorphy of the two quotient spaces is clear as well.

qed

Now we are left with the modification of the definition of trivializations.

Definition 3.2 Let $G$ be a compact topological group, $X$ be a principal $G$-fibre bundle and $\pi: X \longrightarrow X / G$ be the canonical projection. Moreover, let $\mu$ be some normalized measure on $X$.

1. An open $G$-invariant set $U \subseteq X$ is called local trivialization of the principal fibre bundle $X$ iff there is an equivariant homeomorphism $\chi: U \longrightarrow \pi(U) \times(S \backslash G)$ with $\left.\pi\right|_{U}=\operatorname{pr}_{1} \circ \chi$. Here, "equivariant" means $\left(\mathrm{pr}_{2} \circ \chi\right)(x \circ g)=\left(\left(\operatorname{pr}_{2} \circ \chi\right)(x)\right) \cdot[g]_{S}$ for all $x \in U$ and $g \in G$.

We often call $\chi$ itself local trivialization. 
2. A principal fibre bundle is called locally trivial iff there is a covering $\left(U_{\iota}\right)_{\iota \in I}$ of $X$ by local trivializations $U_{\iota}$.

3. A principal fibre bundle is called $\mu$-almost globally trivial iff there is a covering $\left(U_{\iota}\right)$ as in the preceding item where additionally $\mu\left(U_{\iota}\right)=1$ for all $\iota$.

In the following we usually say simply "almost global" instead of " $\mu$ almost global" provided the measure $\mu$ meant is clear.

4. A principal fibre bundle is called globally trivial iff $X$ is a local trivialization.

Here again it should be clear that the definitions above are equivalent to the standard ones. However, we will use these definitions throughout the whole paper when we speak about principal fibre bundles and trivializations.

\section{Principal Fibre Bundle Structure of the Strata}

In order to decide which strata are principal fiber bundles we have to study the form of the stabilizers, i.e. of the base centralizers.

Definition 4.1 The set $\mathbf{B}_{Z}:=\left\{\bar{g} \in \overline{\mathcal{G}} \mid g_{m} \in Z(\mathbf{G})\right.$ and $\left.g_{x}=g_{m} \quad \forall x \in M\right\}$ is called base center.

Lemma 4.1 1 . The base center is contained in every base centralizer.

2. A base centralizer is a normal subgroup of $\overline{\mathcal{G}}$ iff it equals the base center.

3. The base centralizer of a connection equals the base center iff the connection is generic.

Proof Let $\bar{A} \in \overline{\mathcal{A}}$ with base centralizer $\mathbf{B}(\bar{A})$.

1. We have

$$
\begin{aligned}
& \bar{g} \in \mathbf{B}(\bar{A}) \Longleftrightarrow \text { a) } \quad g_{m} \in Z\left(\mathbf{H}_{\bar{A}}\right) \\
& \text { b) } h_{\bar{A}}(\gamma)=g_{m}^{-1} h_{\bar{A}}(\gamma) g_{x} \quad \forall \gamma \in \mathcal{P}_{m x}, x \in M \text {. }
\end{aligned}
$$

Since $Z \subseteq Z(U)$ for all $U \subseteq \mathbf{G}$, we have $\mathbf{B}_{Z} \subseteq \mathbf{B}(\bar{A})$.

2. $\Longrightarrow$ Let $\mathbf{B}(\bar{A})$ be a normal subgroup in $\overline{\mathcal{G}}$.

Let $\bar{g} \in \mathbf{B}(\bar{A})$ and $g_{0} \in \mathbf{G}$. Furthermore, let $x \in M, x \neq m$, and $\gamma \in \mathcal{P}_{m x}$ be arbitrary. Additionally choose a $\bar{g}_{0} \in \overline{\mathcal{G}}$ with $g_{0, m}=g_{0}$ and $g_{0, x}=h_{\bar{A}}(\gamma)$. Then by assumption $\bar{g}_{0}^{-1} \bar{g} \bar{g}_{0} \in \mathbf{B}(\bar{A})$, in particular

$$
\begin{aligned}
h_{\bar{A}}(\gamma) & =g_{0, m}^{-1} g_{m}^{-1} g_{0, m} h_{\bar{A}}(\gamma) g_{0, x}^{-1} g_{x} g_{0, x} \\
& =g_{0}^{-1} g_{m}^{-1} g_{0} h_{\bar{A}}(\gamma) h_{\bar{A}}(\gamma)^{-1} g_{x} h_{\bar{A}}(\gamma) \\
& =g_{0}^{-1} g_{m}^{-1} g_{0} g_{x} h_{\bar{A}}(\gamma),
\end{aligned}
$$

hence $g_{m} g_{0}=g_{0} g_{x}$. Since $g_{0}$ is arbitrary, we have $g_{m}=g_{x}$ for all $x \in M$ and consequently $g_{m} \in Z$. Thus, $\bar{g} \in \mathbf{B}_{Z}$.

We get $\mathbf{B}(\bar{A}) \subseteq \mathbf{B}_{Z}$, hence the equality by the first part of the present proof.

$\Longleftarrow$ Let $\mathbf{B}(\bar{A})=\mathbf{B}_{Z}$. 
Let now $\bar{g} \in \mathbf{B}_{Z}$ and $\bar{g}_{0} \in \overline{\mathcal{G}}$. Then we have $\left(\bar{g}_{0}^{-1} \bar{g} \bar{g}_{0}\right)_{x}=g_{0, x}^{-1} g_{x} g_{0, x}=$ $g_{0, x}^{-1} g_{m} g_{0, x}=g_{0, x}^{-1} g_{0, x} g_{m}=g_{m} \in Z$, hence, in particular, $\left(\bar{g}_{0}^{-1} \bar{g} \bar{g}_{0}\right)_{x}=$ $\left(\bar{g}_{0}^{-1} \bar{g} \bar{g}_{0}\right)_{m}$ for all $x \in M$. Thus, $\bar{g}_{0}^{-1} \bar{g} \bar{g}_{0} \in \mathbf{B}_{Z}$. Hence, $\mathbf{B}(\bar{A})=\mathbf{B}_{Z}$ is a normal subgroup.

3. $\Longrightarrow$ Let $\bar{A} \notin \overline{\mathcal{A}}_{\text {gen }}$, i.e. $Z\left(\mathbf{H}_{\bar{A}}\right) \supset Z$.

Let $g \in Z\left(\mathbf{H}_{\bar{A}}\right) \backslash Z$, and set $\bar{g}:=\left(h_{\bar{A}}\left(\gamma_{x}\right)^{-1} g h_{\bar{A}}\left(\gamma_{x}\right)\right)_{x \in M}$ with $\gamma_{x}$ chosen as usual. Per definitionem we have $\bar{g} \in \mathbf{B}(\bar{A})$, but $\bar{g} \notin \mathbf{B}_{Z}$.

$\Longleftarrow$ Let $\bar{A} \in \overline{\mathcal{A}}_{\text {gen }}$, i.e. $Z\left(\mathbf{H}_{\bar{A}}\right)=Z$. Now we have

$$
\begin{aligned}
& \bar{g} \in \mathbf{B}(\bar{A}) \Longleftrightarrow \text { a) } g_{m} \in Z\left(\mathbf{H}_{\bar{A}}\right)=Z \\
& \text { b) } h_{\bar{A}}(\gamma)=g_{m}^{-1} h_{\bar{A}}(\gamma) g_{x} \quad \forall \gamma \in \mathcal{P}_{m x}, x \in M \\
& \Longleftrightarrow \text { a) } g_{m} \in Z \\
& \text { b) } h_{\bar{A}}(\gamma)=h_{\bar{A}}(\gamma) g_{m}^{-1} g_{x} \quad \forall \gamma \in \mathcal{P}_{m x}, x \in M \\
& \Longleftrightarrow \text { a) } g_{m} \in Z \\
& \text { b) } g_{m}=g_{x} \quad \forall x \in M \\
& \Longleftrightarrow \bar{g} \in \mathbf{B}_{Z} \text {. }
\end{aligned}
$$

qed

Consequently, only the generic stratum is a principal fibre bundle:

Proposition 4.2 Let $t \in \mathcal{T}$ be a gauge orbit type. Then we have:

The stratum $\overline{\mathcal{A}}_{=t}$ is a principal fibre bundle iff $t=t_{\text {max }}$, i.e. $\overline{\mathcal{A}}_{=t}=\overline{\mathcal{A}}_{\text {gen }}$.

Proof By the definition of the gauge orbit type, all holonomy centralizers $Z\left(\mathbf{H}_{\bar{A}}\right)$ occurring in a fixed stratum $\overline{\mathcal{A}}_{=t}$ are conjugate. As proven in 13 the same is true for the stabilizers $\mathbf{B}(\bar{A})$.

Now $\overline{\mathcal{A}}_{=t}$ is a principal fibre bundle iff all connections in $\overline{\mathcal{A}}_{=t}$ have the same stabilizer. On the other hand, this is true iff this stabilizer is a normal subgroup in $\overline{\mathcal{G}}$. The lemma above yields the assertion.

qed

\section{$5 \quad$ Almost Global Triviality of $\left(\mathbf{G}^{k}\right)_{\text {gen }}$}

In Section 7 we will prove that $\overline{\mathcal{A}}_{\text {gen }}$ is not only a locally trivial, but even an almost globally trivial principal fibre bundle. As mentioned in the introduction we will deduce this by means of the reduction mapping from the corresponding statement on the generic stratum of $\mathbf{G}^{k}$ w.r.t. the adjoint action that we are going to deal with in the present section.

Definition 5.1 Let $k \in \mathbb{N}_{+}$.

An element $\vec{g}:=\left(g_{1}, \ldots, g_{k}\right) \in \mathbf{G}^{k}$ (and its orbit, resp.) is called generic iff $\operatorname{Typ}(\vec{g}) \equiv\left[Z\left(\left\{g_{1}, \ldots, g_{k}\right\}\right)\right]=[Z]$.

The set of all generic elements of $\mathbf{G}^{k}$ is denoted by $\left(\mathbf{G}^{k}\right)_{\text {gen }}$.

Since $\operatorname{Typ}(\vec{g}) \leq[Z]$ for all $\vec{g} \in \mathbf{G}^{k}$, every generic orbit is of maximal type. Moreover, a Lie group is abelian iff one (and then each) (nontrivial) power consists of generic elements only.

\footnotetext{
${ }^{1}$ Let $G$ be a group acting on $X$ where all stabilizers $G_{x}, x \in X$, are conjugate. If $X$ is a principal fibre bundle, then $G_{x}=G_{y}$ for all $x, y \in X$ and, in particular, $G_{x}=G_{x \circ g}=g^{-1} G_{x} g$ for all $g$. Thus, $G_{x}$ is a normal subgroup in $G$. Conversely, let all $G_{x}$ be normal subgroups. Then, since all stabilizers are conjugate, we have $G_{y}=g^{-1} G_{x} g \subseteq G_{x}$ for all $x, y \in X$, i.e., $X$ is a principal fibre bundle.

${ }^{2}$ We have $Z\left(\vec{e}_{\mathbf{G}}\right):=Z\left(\left(e_{\mathbf{G}}, \ldots, e_{\mathbf{G}}\right)\right)=\mathbf{G}$. If $\mathbf{G}^{k}$ now consists for $k \in \mathbb{N}_{+}$of generic elements only, then $Z=Z\left(\vec{e}_{\mathbf{G}}\right)=\mathbf{G}$. Conversely, if $Z=\mathbf{G}$, then $\mathbf{G} \supseteq Z(\vec{g}) \supseteq Z=\mathbf{G}$, hence $Z(\vec{g})=Z$ for all $\vec{g} \in \mathbf{G}^{k}$.
} 
Proposition 5.1 There is a $k_{\text {min }} \in \mathbb{N}_{+}$, such that the generic stratum $\left(\mathbf{G}^{k}\right)_{\text {gen }}$ is an open and dense submanifold of $\mathbf{G}^{k}$ with full Haar measure for all $k \geq k_{\min }$ and is empty for all $k<k_{\text {min }}$.

Proof 1. Choice of $k_{\min }$

By the finiteness lemma for centralizers [13 there is a $k \in \mathbb{N}_{+}$, such that there is at least one orbit in $\mathbf{G}^{k}$ having type $[Z(\mathbf{G})]$. Now, choose for $k_{\min }$ simply the minimum of all such $k$.

- Obviously $\left(\mathbf{G}^{k}\right)_{\text {gen }}=\varnothing$ for $k<k_{\min }$.

- For $k \geq k_{\min }$ there is at least one generic element in $\mathbf{G}^{k}$.

Namely, let $\vec{g}:=\left(g_{1}, \ldots, g_{k_{\min }}\right) \in \mathbf{G}^{k_{\min }}$ be a generic element. Then $\vec{g}_{k}:=$ $\left(g_{1}, \ldots, g_{k_{\min }}, e_{\mathbf{G}}, \ldots, e_{\mathbf{G}}\right) \in \mathbf{G}^{k}$ is generic as well because of

$$
\begin{aligned}
{[Z] } & \geq \operatorname{Typ}\left(\vec{g}_{k}\right)=\left[Z\left(\left\{g_{1}, \ldots, g_{k_{\min }}, e_{\mathbf{G}}, \ldots, e_{\mathbf{G}}\right\}\right)\right] \\
& =\left[Z\left(\left\{g_{1}, \ldots, g_{k_{\min }}\right\}\right)\right]=\operatorname{Typ}(\vec{g})=[Z] .
\end{aligned}
$$

Let now $k \geq k_{\min }$ in the following.

2. $\left(\mathbf{G}^{k}\right)_{\text {gen }}$ is a smooth submanifold of $\mathbf{G}^{k}$, since the adjoint action is smooth. [6]

3. $\left(\mathbf{G}^{k}\right)_{\text {gen }}$ is open, since any of its points possesses an open neighbourhood whose points have at least, hence by the maximality of $[Z]$ even exactly type $[Z]$. [6]

4. Since $\mathbf{G}$ is connected, $\mathbf{G}^{k} / \mathrm{Ad}$ is connected again. Again by the maximality of $[Z]$, we have $\overline{\left(\mathbf{G}^{k}\right)_{\text {gen }}} \equiv \overline{\left(\mathbf{G}^{k}\right)_{Z}}=\bigcup_{[\mathbf{K}] \leq[Z]}\left(\mathbf{G}^{k}\right)_{\mathbf{K}}=\mathbf{G}^{k}$. [26] Here we set $\left(\mathbf{G}^{k}\right)_{\mathbf{K}}=\left\{\vec{g} \in \mathbf{G}^{k} \mid \operatorname{Typ}(\vec{g})=[\mathbf{K}]\right\}$.

5. Now, $\left(\mathbf{G}^{k}\right)_{\mathbf{H}}$ is a smooth submanifold of $\mathbf{G}^{k}$ for every closed subgroup $\mathbf{H}$ of $\mathbf{G}$ with $[\mathbf{H}]<[Z]$. [26] Moreover, $\operatorname{dim}\left(\mathbf{G}^{k}\right)_{\mathbf{H}}<\operatorname{dim}\left(\mathbf{G}^{k}\right)_{Z} \equiv \operatorname{dim}\left(\mathbf{G}^{k}\right)_{\text {gen }}=\operatorname{dim} \mathbf{G}^{k}$. Since the Haar measure of a lower-dimensional submanifold vanishes [9, 10], we have $\mu_{\text {Haar }}\left(\left(\mathbf{G}^{k}\right)_{\mathbf{H}}\right)=0$. Since there are only finitely many orbit types on $\mathbf{G}^{k}$ [26], we get $\mu_{\text {Haar }}\left(\mathbf{G}^{k} \backslash\left(\mathbf{G}^{k}\right)_{\text {gen }}\right)=\mu_{\text {Haar }}\left(\bigcup_{[\mathbf{H}]<[Z]}\left(\mathbf{G}^{k}\right)_{\mathbf{H}}\right)=0 . \quad$ qed

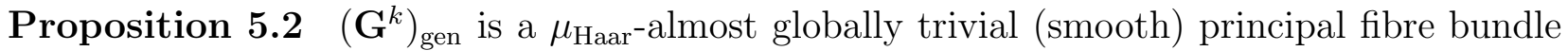
having structure group $Z \backslash \mathbf{G}$ for all $k \geq k_{\min }$.

Proof 1. Bundle structure over $\left(\mathbf{G}^{k}\right)_{\text {gen }} / \mathrm{Ad}$

The adjoint action of $\mathbf{G}$ on $\mathbf{G}^{k}$ is smooth. Hence the generic stratum $\left(\mathbf{G}^{k}\right)_{\text {gen }} \equiv$ $\left(\mathbf{G}^{k}\right)_{Z}$ is a smooth submanifold and $\pi_{k}:\left(\mathbf{G}^{k}\right)_{\text {gen }} \longrightarrow\left(\mathbf{G}^{k}\right)_{\text {gen }} /$ Ad is a smooth (locally trivial) fibre bundle with typical fibre and structure group $Z \backslash \mathbf{G}$ ( 6 and note $N(Z)=\mathbf{G}$ ). Since the stabilizer of any point in $\left(\mathbf{G}^{k}\right)_{\text {gen }}$ equals $Z, \pi_{k}$ is (in our notation) even a principal fibre bundle over the generic stratum.

2. Choice of a neighbourhood $V_{k, \vec{v}}$ for every fixed $\vec{v} \in\left(\mathbf{G}^{k}\right)_{\text {gen }}$

First we cut out from the $n$-dimensional smooth manifold $\left(\mathbf{G}^{k}\right)_{\text {gen }} / \mathrm{Ad}$ a neighbourhood $B^{n}$ of $[\vec{v}]$ diffeomorphic to an $n$-dimensional ball and get a remaining smooth manifold $N$. By general arguments [24, 29, 28] there are a simplicial complex $K$ consisting of countably many simplices and a smooth triangulation $f: K \longrightarrow N$. Let $K_{n}$ denote the set of all $n$-cells s] $^{3}$ of . It can be constructed from $K$ by deleting the $(n-1)$-skeleton $K_{\leq n-1}$, i.e. all cells whose dimension is smaller than that of $K$. Now define $V_{k, \vec{v}}:=\pi_{k}^{-1}\left(f\left(K_{n}\right) \cup \operatorname{int} B^{n}\right)$.

\footnotetext{
${ }^{3}$ Note that here (in contrast to the standard definition) a cell $\widehat{\sigma}$ is the interior of a simplex $\sigma$. Only for dimension 0 the cell shall be a simplex.
} 
3. Properties of $V_{k, \vec{v}}$

- $V_{k, \vec{v}}$ is Ad-invariant.

- $\vec{v} \in V_{k, \vec{v}}$.

- Since $f$ is a smooth triangulation, $f\left(K_{n}\right)$ is a smooth manifold [9] that equals the disjoint union of all $f(\widehat{\sigma})$ where $\sigma$ is an $n$-simplex of $K$. Since $f$ is a homeomorphism and $\widehat{\sigma}$ always contractible, also $f(\widehat{\sigma})$ is always contractible. The contractiblity of int $B^{n}$ is trivial. Moreover, int $B^{n}$ and $f\left(K_{n}\right)$ are disjoint.

- Hence, as a $\pi_{k}$-preimage of a submanifold of $\left(\mathbf{G}^{k}\right)_{\operatorname{gen}} /$ Ad the set $V_{k, \vec{v}}$ is a submanifold of $\left(\mathbf{G}^{k}\right)_{\text {gen }}$, thus of $\mathbf{G}^{k}$ as well. In particular, $V_{k, \vec{v}}$ is open in $\mathbf{G}^{k}$ by the continuity of $\pi_{k}$. Thus $\pi_{k}\left(V_{k, \vec{v}}\right)$ is a disjoint union of contractible manifolds.

- We have $V_{k, \vec{v}}=\left(\mathbf{G}^{k}\right)_{\text {gen }} \backslash\left(\pi_{k}^{-1}\left(\partial B^{n}\right) \cup \bigcup_{\sigma \in K_{\leq n-1}} \pi_{k}^{-1}(f(\widehat{\sigma}))\right)$, i.e. $V_{k, \vec{v}}$ emerges from $\left(\mathbf{G}^{k}\right)_{\text {gen }}$ by deleting the $\pi_{k}$-preimages of the boundary of $B^{n}$ and of all images of lower-dimensional skeletons in $\left(\mathbf{G}^{k}\right)_{\text {gen }} / \mathrm{Ad}$, respectively.

- We have $\mu_{\text {Haar }}\left(V_{k, \vec{v}}\right)=1$.

Since $\mu_{\text {Haar }}\left(\left(\mathbf{G}^{k}\right)_{\text {gen }}\right)=1$, it is sufficient to prove that the just eliminated objects have Haar measure 0 .

a) Let $\sigma \in K_{\leq n-1}$. Since $f$ is a homeomorphism, we have $\operatorname{dim} f(\widehat{\sigma})=$ $\operatorname{dim} \widehat{\sigma}<n$. As above $\pi_{k}^{-1}(f(\widehat{\sigma}))$ is a submanifold of $\left(\mathbf{G}^{k}\right)_{\text {gen }}$, hence also of $\mathbf{G}^{k}$, whose codimension is $n-\operatorname{dim} \hat{\sigma}>0$. Thus, this set is a zero set, too.

Together with $K$, obviously also $K_{\leq n-1}$ is a complex with countably many simplices. Hence,

$$
\mu_{\text {Haar }}\left(\bigcup_{\sigma \in K_{\leq n-1}} \pi_{k}^{-1}(f(\widehat{\sigma}))\right) \leq \sum_{\sigma \in K_{\leq n-1}} \mu_{\text {Haar }}\left(\pi_{k}^{-1}(f(\widehat{\sigma}))\right)=0 .
$$

b) $\partial B^{n}$ is a smooth submanifold of $\left(\mathbf{G}^{k}\right)_{\operatorname{gen}} /$ Ad with codimension 1 . Thus, $\pi_{k}^{-1}\left(\partial B^{n}\right)$ is a smooth submanifold of $\left(\mathbf{G}^{k}\right)_{\text {gen }}$ with codimension 1. Again, $\mu_{\text {Haar }}\left(\pi_{k}^{-1}\left(\partial B^{n}\right)\right)=0$.

- $V_{k, \vec{v}}$ is dense in $\mathbf{G}^{k}$. This follows from $\mu_{\text {Haar }}\left(V_{k, \vec{v}}\right)=1$ and the strict positivity of the Haar measure because $V_{k, \vec{v}}$ is open.

4. Triviality of $\left.\pi_{k}\right|_{V_{k, \vec{v}}}$ $\left.\pi_{k}\right|_{V_{k, \vec{v}}}: V_{k, \vec{v}} \longrightarrow \pi_{k}\left(V_{k, \vec{v}}\right)=\operatorname{int} B^{n} \cup f\left(K_{n}\right)$ is a principal fibre bundle over the disjoint union int $B^{n} \cup f\left(K_{n}\right)$ of contractible manifolds having structure group $Z \backslash$ G. Hence [18], this bundle is trivial, i.e., there is an equivariant homeomorphism $V_{k, \vec{v}} \cong \pi\left(V_{k, \vec{v}}\right) \times(Z \backslash \mathbf{G})$.

5. Almost global triviality of $\left(\mathbf{G}^{k}\right)_{\text {gen }}$

Obviously $\mathcal{V}_{k}:=\left\{V_{k, \vec{v}} \mid \vec{v} \in\left(\mathbf{G}^{k}\right)_{\text {gen }}\right\}$ is a non-empty covering of $\left(\mathbf{G}^{k}\right)_{\text {gen }}$ by $\mu_{\text {Haar-almost global trivializations. }}$

qed

\section{Relations between the Structure Groups}

We know already that the structure group of the principal fibre bundle $\left(\mathbf{G}^{k}\right)_{\text {gen }}$ equals $Z \backslash \mathbf{G}$ and that of the principal fibre bundle $\overline{\mathcal{A}}_{\text {gen }}$ equals $\mathbf{B}_{Z} \backslash \overline{\mathcal{G}}$. In order to lift the almost global triviality of $\left(\mathbf{G}^{k}\right)_{\text {gen }}$ to that of $\overline{\mathcal{A}}_{\text {gen }}$ in the next section, we have to investigate the relation between the corresponding structure groups. We need 
Definition 6.1 Let $*:(Z \backslash \mathbf{G}) \times \overline{\mathcal{G}} \longrightarrow \mathbf{B}_{Z} \backslash \overline{\mathcal{G}}$ be defined by $[g]_{Z} * \bar{g}:=\left[\left(g g_{x}\right)_{x \in M}\right]_{\mathbf{B}_{Z}}$.

Lemma $6.1 \bullet *$ is well-defined and continuous.

- The restriction of $*$ to $(Z \backslash \mathbf{G}) \times \overline{\mathcal{G}}_{0}$ is an isomorphism.

We recall $\overline{\mathcal{G}}_{0}:=\left\{\bar{g} \in \overline{\mathcal{G}} \mid g_{m}=e_{\mathbf{G}}\right\}$.

Proof $\bullet$ Let $g_{1} \sim g_{2}$, i.e. $g_{1}=z g_{2}$ for a $z \in Z$. Then we have

$$
\begin{aligned}
{\left[g_{1}\right]_{Z} * \bar{g} } & =\left[\left(g_{1} g_{x}\right)_{x \in M}\right]_{\mathbf{B}_{Z}} \\
& =\left[\left(z g_{2} g_{x}\right)_{x \in M}\right]_{\mathbf{B}_{Z}} \\
& =\left[\bar{z} \circ\left(g_{2} g_{x}\right)_{x \in M}\right]_{\mathbf{B}_{Z}} \quad\left(\bar{z} \equiv(z)_{x \in M} \in \mathbf{B}_{Z}\right) \\
& =\left[\left(g_{2} g_{x}\right)_{x \in M}\right]_{\mathbf{B}_{Z}} \\
& =\left[g_{2}\right]_{Z} * \bar{g} .
\end{aligned}
$$

- The continuity of $*$ follows immediately from the surjectivity and openness of the canonical projection $\mathbf{G} \times \overline{\mathcal{G}} \longrightarrow(Z \backslash \mathbf{G}) \times \overline{\mathcal{G}}$, the continuity of $\mathbf{G} \times \overline{\mathcal{G}} \longrightarrow \overline{\mathcal{G}}$ with $(g, \bar{g}) \longmapsto\left(g g_{x}\right)_{x \in M}$ as well as that of the canonical projection $\overline{\mathcal{G}} \longrightarrow \mathbf{B}_{Z} \backslash \overline{\mathcal{G}}$ and the commutativity of the corresponding diagram

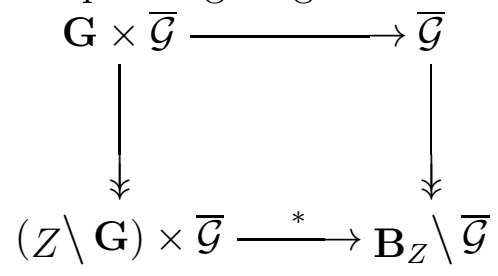

- $*$ is injective.

Let $\left[g_{1}\right]_{Z} * \bar{g}_{1}=\left[g_{2}\right]_{Z} * \bar{g}_{2}$ with $\bar{g}_{1}, \bar{g}_{2} \in \overline{\mathcal{G}}_{0}$, i.e. $g_{1, m}=g_{2, m}=e_{\mathbf{G}}$. Then per def. $\left[\left(g_{1} g_{1, x}\right)_{x \in M}\right]_{\mathbf{B}_{Z}}=\left[\left(g_{2} g_{2, x}\right)_{x \in M}\right]_{\mathbf{B}_{Z}}$. Thus, there is a $z \in Z$ with $g_{1} g_{1, x}=z g_{2} g_{2, x}$ for all $x \in M$. For $x=m$ we have $g_{1}=z g_{2}$ (and so $\left[g_{1}\right]_{Z}=\left[g_{2}\right]_{Z}$ ) and consequently $\bar{g}_{1}=\bar{g}_{2}$.

- $*$ is surjective.

Let $[\bar{g}]_{\mathbf{B}_{Z}}$ be given. Choose a representative $\bar{g}^{\prime} \in[\bar{g}]_{\mathbf{B}_{Z}}$. Set $g^{\prime \prime}:=g_{m}^{\prime}$ and $g_{x}^{\prime \prime}:=$ $\left(g^{\prime \prime}\right)^{-1} g_{x}^{\prime}$. Then $\left[g^{\prime \prime}\right]_{Z} * \bar{g}^{\prime \prime}=[\bar{g}]_{\mathbf{B}_{Z}}$.

qed

The map * is a modification of the isomorphism $\Psi_{0}: \overline{\mathcal{G}}_{0} \times Z\left(\mathbf{H}_{\bar{A}}\right) \backslash \mathbf{G} \longrightarrow \mathbf{B}(\bar{A}) \backslash \overline{\mathcal{G}}$ [13], tailored to the special case of generic connections. There we started with the canonical isomorphism $\left.\phi^{\prime}\right|_{Z\left(\mathbf{H}_{\bar{A}}\right)}=\phi^{-1}: Z\left(\mathbf{H}_{\bar{A}}\right) \longrightarrow \mathbf{B}(\bar{A}), g \longmapsto\left(h_{\bar{A}}\left(\gamma_{x}\right)^{-1} g h_{\bar{A}}\left(\gamma_{x}\right)\right)_{x \in M}$ and extended it in a natural way to the whole $\mathbf{G}$. For generic connections, $\left.\phi^{\prime}\right|_{Z}$ now maps $g$ simply on $\left(h_{\bar{A}}\left(\gamma_{x}\right)^{-1} g h_{\bar{A}}\left(\gamma_{x}\right)\right)_{x \in M}=(g)_{x \in M}$. But, therewith we get a second natural extension of $\left.\phi^{\prime}\right|_{Z}$ to the whole $\mathbf{G}$. This is just the definition of $*$ above.

\section{Almost Global Triviality of $\overline{\mathcal{A}}_{\text {gen }}$}

We start with

Proposition 7.1 $\overline{\mathcal{A}}_{\text {gen }}$ has the induced Haar measure 1.

Proof By Proposition 5.1, $\left(\mathbf{G}^{k}\right)_{\text {gen }}$ has Haar measure 1 for some $k \in \mathbb{N}_{+}$. Now, let $\Gamma$ be some graph with $k$ edges $\alpha_{i}$ and the vertex $m$. Since the corresponding reduction 
mapping $\varphi \equiv \pi_{\Gamma}: \overline{\mathcal{A}} \longrightarrow \mathbf{G}^{k}$ decreases the type, [13 we have $[Z]=\operatorname{Typ}(\varphi(\bar{A})) \leq$ $\operatorname{Typ}(\bar{A}) \leq[Z]$ for all $\bar{A} \in \varphi^{-1}\left(\left(\mathbf{G}^{k}\right)_{\text {gen }}\right)$. Hence $\overline{\mathcal{A}}_{\text {gen }} \supseteq \varphi^{-1}\left(\left(\mathbf{G}^{k}\right)_{\text {gen }}\right)$. By definition of the induced Haar measure,

$$
1 \geq \mu_{0}\left(\overline{\mathcal{A}}_{\text {gen }}\right) \geq \mu_{0}\left(\varphi^{-1}\left(\left(\mathbf{G}^{k}\right)_{\text {gen }}\right)\right)=\mu_{\text {Haar }}\left(\left(\mathbf{G}^{k}\right)_{\text {gen }}\right)=1 . \quad \text { qed }
$$

The goal of the present section is the proof of the following

Theorem 7.2 $\overline{\mathcal{A}}_{\text {gen }}$ is (w.r.t. the action of $\overline{\mathcal{G}}$ ) a $\mu_{0}$-almost globally trivial principal fibre bundle with structure group $\mathbf{B}_{Z} \backslash \overline{\mathcal{G}}$.

\subsection{Independent Generators of the Holonomy Centralizer}

Our task is to find for all $\bar{A} \in \overline{\mathcal{A}}_{\text {gen }}$ a neighbourhood $U_{\bar{A}}$ that, on the one hand, has the full measure 1 and, on the other hand, is a local trivialization of $\overline{\mathcal{A}}_{\text {gen }}$. For that purpose we choose for $\bar{A}$ according to the finiteness lemma for centralizers [13 finitely many paths $\alpha_{i}$ in $\mathcal{H} \mathcal{G}$ with $Z\left(h_{\bar{A}}(\boldsymbol{\alpha})\right)=Z\left(\mathbf{H}_{\bar{A}}\right)=Z$ and denote the corresponding reduction mapping shortly by $\varphi$. The most obvious choice of a neighbourhood of $\bar{A}$ would be $U:=\varphi^{-1}(V)$, where $V$ is an almost global trivialization of $\left(\mathbf{G}^{k}\right)_{\text {gen }}$ from the covering above with $\varphi(\bar{A}) \in V$. But, it can happen that despite of $\mu_{\text {Haar }}(V)=1$ the induced Haar measure of $U$ is smaller than 1. This is, in particular, the case if $\varphi$ is not surjective, because, e.g., the paths in $\boldsymbol{\alpha}$ are not independent or one path in $\boldsymbol{\alpha}$ occurs twice what is not a priori forbidden. That is why we have to guarantee that we can always find an $\boldsymbol{\alpha}$ fulfilling a certain independency condition. For that purpose we will need the notion of hyphs [12]: A hyph $v$ is a set of "independent" paths. For instance, graphs and webs [4] are special hyphs. One of the most striking properties of a hyph is that the parallel transports can be assigned to the paths independently. Moreover, the corresponding projection $\pi_{v}: \overline{\mathcal{A}} \longrightarrow \mathbf{G}^{\# v}, \bar{A} \longrightarrow h_{\bar{A}}(v)$, gives $\mu_{0}\left(\pi_{v}^{-1}(W)\right)=\mu_{\text {Haar }}(W)$ for all $W$.

It would be optimal if we were able to show that for every $\bar{A}$ there is a hyph $\boldsymbol{\alpha}$ with $Z\left(\mathbf{H}_{\bar{A}}\right)=$ $Z\left(h_{\bar{A}}(\boldsymbol{\alpha})\right)$. However, though we can find - starting with an arbitrary $\boldsymbol{\beta}$ with $Z\left(\mathbf{H}_{\bar{A}}\right)=$ $Z\left(h_{\bar{A}}(\boldsymbol{\beta})\right)$ - a hyph $v$ such that all paths in $\boldsymbol{\beta}$ can be written as products of paths in $v$, this set $v$ typically consists not only of paths in $\mathcal{H G}$, i.e. closed paths only. To avoid this problem we weaken the notion of a hyph.

Definition 7.1 A finite set $\boldsymbol{\alpha} \subseteq \mathcal{H G}$ is called weak hyph iff the corresponding reduction mapping

is surjective and fulfills

$$
\begin{aligned}
\varphi_{\boldsymbol{\alpha}}: \overline{\mathcal{A}} & \longrightarrow \mathbf{G}^{\# \boldsymbol{\alpha}} \\
\bar{A}^{\prime} & \longmapsto h_{\bar{A}^{\prime}}(\boldsymbol{\alpha})
\end{aligned}
$$

$$
\mu_{0}\left(\pi_{\boldsymbol{\alpha}}^{-1}(W)\right)=\mu_{\text {Haar }}(W) \text { for all } W \subseteq \mathbf{G}^{\# \boldsymbol{\alpha}} .
$$

Obviously, every hyph that consists only of closed paths is a weak hyph.

Proposition 7.3 For every $\bar{A} \in \overline{\mathcal{A}}$ there is a weak hyph $\boldsymbol{\alpha} \subseteq \mathcal{H} \mathcal{G}$ with $Z\left(h_{\bar{A}}(\boldsymbol{\alpha})\right)=Z\left(\mathbf{H}_{\bar{A}}\right)$.

The proof of this proposition is very technical and is therefore shifted to Appendix $\mathbb{F}$. 


\subsection{Choice of the Covering of $\overline{\mathcal{A}}_{\text {gen }}$}

Now, we are able to define the desired covering of $\overline{\mathcal{A}}_{\text {gen }}$ : For each $\bar{A} \in \overline{\mathcal{A}}_{\text {gen }}$ we choose a weak hyph $\boldsymbol{\alpha} \subseteq \mathcal{H} \mathcal{G}$ with $Z\left(h_{\bar{A}}(\boldsymbol{\alpha})\right)=Z\left(\mathbf{H}_{\bar{A}}\right)=Z$ by Proposition 7.3 and denote the corresponding reduction mapping as usual by $\varphi_{\boldsymbol{\alpha}}$. Now we choose according to Section 5 an almost global trivialization $V_{\# \boldsymbol{\alpha}, \varphi_{\boldsymbol{\alpha}}(\bar{A})}$ of $\left(\mathbf{G}^{\# \boldsymbol{\alpha}}\right)_{\text {gen }}$ that contains $\varphi_{\boldsymbol{\alpha}}(\bar{A})$, and set $U_{\bar{A}}:=\varphi_{\boldsymbol{\alpha}}^{-1}\left(V_{\# \boldsymbol{\alpha}, \varphi_{\boldsymbol{\alpha}}(\bar{A})}\right) \subseteq \overline{\mathcal{A}}$. By $\bar{A} \in U_{\bar{A}}$ we have

Proposition 7.4 $\mathcal{U}:=\left\{U_{\bar{A}}\right\}_{\bar{A} \in \overline{\mathcal{A}}_{\text {gen }}}$ is a covering of $\overline{\mathcal{A}}_{\text {gen }}$.

\subsection{Properties of the Covering}

We still have to show that each $U_{\bar{A}}$ is an almost global trivialization of $\overline{\mathcal{A}}_{\text {gen }}$. For this, let us fix an arbitrary $\bar{A} \in \overline{\mathcal{A}}_{\text {gen }}$ with reduction mapping $\varphi:=\varphi_{\boldsymbol{\alpha}}$ and set simply $k:=\# \boldsymbol{\alpha}$, $V:=V_{k, \varphi(\bar{A})} \subseteq\left(\mathbf{G}^{k}\right)_{\text {gen }}$ and $U:=U_{\bar{A}}=\varphi^{-1}(V)$.

The easily verifiable properties of $U$ are described by

Lemma 7.5 $U$ is an open, dense, $\overline{\mathcal{G}}$-invariant subset of $\overline{\mathcal{A}}_{\text {gen }}$ with $\mu_{0}(U)=1$.

Proof By construction, $V$ is an open, dense, Ad-invariant subset of $\left(\mathbf{G}^{k}\right)_{\text {gen }}$ with $\mu_{\text {Haar }}(V)=$ 1. Since $\varphi$ always preserves or reduces the types, [13] we have $[Z]=\operatorname{Typ}\left(\varphi\left(\bar{A}^{\prime}\right)\right) \leq$ $\operatorname{Typ}\left(\bar{A}^{\prime}\right) \leq[Z]$, i.e. $\bar{A}^{\prime} \in \overline{\mathcal{A}}_{\text {gen }}$ for all $\bar{A}^{\prime} \in U$. Since $\varphi$ is continuous, $U=\varphi^{-1}(V)$ is open as a subset of $\overline{\mathcal{A}}$, hence as a subset of $\overline{\mathcal{A}}_{\text {gen }}$ as well due to the openness of $\overline{\mathcal{A}}_{\text {gen }}$. Since $\boldsymbol{\alpha}$ is a weak hyph, we have $\mu_{0}(U)=\mu_{0}\left(\varphi^{-1}(V)\right)=\mu_{\text {Haar }}(V)=1$ by Proposition 7.3. Due to the strict positivity of $\mu_{0}$, both statements yield the denseness of $U$ in $\overline{\mathcal{A}}_{\text {gen }}$. Finally, the $\overline{\mathcal{G}}$-invariance of $U$ follows from the Ad-invariance of $V$.

qed

The most important property of $U$, however, requires a longer proof.

Proposition 7.6 $U$ is a local trivialization of $\overline{\mathcal{A}}_{\text {gen }}$.

Proof We denote the equivariant homeomorphism that belongs to the almost global trivialization of $\mathbf{G}^{k}$ according Proposition 5.2 by $\psi: V \longrightarrow \pi_{k}(V) \times(Z \backslash \mathbf{G})$. The projection onto the second component be $\psi_{2}: V \longrightarrow Z \backslash \mathbf{G}$. Furthermore, let $\gamma_{x}$ be for every $x \in M$ some fixed path from $m$ to $x$. W.l.o.g., $\gamma_{m}$ is the trivial path.

Now we define the trivialization mapping of $\overline{\mathcal{A}}$ :

$$
\begin{array}{ccc}
\Psi: U & \longrightarrow(U) \times \mathbf{B}_{Z} \backslash \overline{\mathcal{G}} . \\
h & \longmapsto\left([h], \psi_{2}(\varphi(h)) *\left(h\left(\gamma_{x}\right)\right)_{x \in M}\right)
\end{array}
$$

1. $\Psi$ is well-defined.

Because of $h \in U$ we have $\varphi(h) \in V$, i.e. $\psi_{2}(\varphi(h))$ is well-defined.

2. $\Psi$ is surjective.

Let $\left([h],[\bar{g}]_{\mathbf{B}_{Z}}\right) \in \pi(U) \times \mathbf{B}_{Z} \backslash \overline{\mathcal{G}}$ be given. By Lemma 6.1 there is exactly one $\left[g^{\prime}\right]_{Z} \in Z \backslash \mathbf{G}$ and some $\bar{g}^{\prime} \in \overline{\mathcal{G}}_{0}$ with $\left[g^{\prime}\right]_{Z} * \bar{g}^{\prime}=[\bar{g}]_{\mathbf{B}_{Z}}$. Additionally choose some $h^{\prime} \in[h]$. Hence, $h^{\prime} \in U$ and $\varphi\left(h^{\prime}\right) \equiv h^{\prime}(\boldsymbol{\alpha}) \in V$. Let $\vec{g}:=\psi^{-1}\left(\left[h^{\prime}(\boldsymbol{\alpha})\right]_{\mathrm{Ad}},\left[g^{\prime}\right]_{Z}\right)$. Since $h^{\prime}(\boldsymbol{\alpha})$ and $\vec{g}$ are in one and the same orbit w.r.t. the adjoint action, there is a $\widetilde{g} \in \mathbf{G}$ with $\vec{g}=\widetilde{g}^{-1} h^{\prime}(\boldsymbol{\alpha}) \widetilde{g}$. Now, let $\bar{h}(\gamma):=\left(g_{x}^{\prime}\right)^{-1} \widetilde{g}^{-1} h^{\prime}\left(\gamma_{x} \gamma \gamma_{y}^{-1}\right) \widetilde{g} g_{y}^{\prime}$ for all $\gamma \in \mathcal{P}_{x y}$. 
Obviously, $\bar{h}$ is gauge equivalent to $h^{\prime}$ by means of the gauge transform $\left(h^{\prime}\left(\gamma_{x}\right)^{-1} \widetilde{g} g_{x}^{\prime}\right)_{x \in M}$. Hence $[\bar{h}]=\left[h^{\prime}\right]=[h]$ and $\bar{h} \in U$. Moreover, $\varphi(\bar{h}) \equiv \bar{h}(\boldsymbol{\alpha})=$ $\widetilde{g}^{-1} h^{\prime}(\boldsymbol{\alpha}) \widetilde{g}$. Finally,

$$
\begin{aligned}
\Psi(\bar{h}) & =\left([\bar{h}], \psi_{2}(\varphi(\bar{h})) *\left(\bar{h}\left(\gamma_{x}\right)\right)_{x \in M}\right) \\
& =\left([h], \psi_{2}\left(\widetilde{g}^{-1} h^{\prime}(\boldsymbol{\alpha}) \widetilde{g}\right) *\left(\left(g_{m}^{\prime}\right)^{-1} \widetilde{g}^{-1} h^{\prime}(1) \widetilde{g} g_{x}^{\prime}\right)_{x \in M}\right) \\
& =\left([h], \psi_{2}(\vec{g}) *\left(g_{x}^{\prime}\right)_{x \in M}\right) \\
& =\left([h],\left[g^{\prime}\right]_{Z} * \bar{g}^{\prime}\right) \\
& =\left([h],[\bar{g}]_{\mathbf{B}_{Z}}\right) .
\end{aligned}
$$

3. $\Psi$ is injective.

Let $\Psi\left(h_{1}\right)=\Psi\left(h_{2}\right)$. Then, in particular, $\psi_{2}\left(\varphi\left(h_{1}\right)\right) *\left(h_{1}\left(\gamma_{x}\right)\right)_{x \in M}=\psi_{2}\left(\varphi\left(h_{2}\right)\right) *$ $\left(h_{2}\left(\gamma_{x}\right)\right)_{x \in M}$. From the bijectivity of $*$ on $(Z \backslash \mathbf{G}) \times \overline{\mathcal{G}}_{0}$ we get $h_{1}\left(\gamma_{x}\right)=h_{2}\left(\gamma_{x}\right)$ for all $x \in M$ and $\psi_{2}\left(h_{1}(\boldsymbol{\alpha})\right)=\psi_{2}\left(h_{2}(\boldsymbol{\alpha})\right)$. Since by assumption $h_{1}$ and $h_{2}$ are gauge equivalent, there is a $\bar{g} \in \overline{\mathcal{G}}$ with $h_{1}=h_{2} \circ \bar{g}$. In particular, we have $h_{1}(\boldsymbol{\alpha})=g_{m}^{-1} h_{2}(\boldsymbol{\alpha}) g_{m}$, i.e., $h_{1}(\boldsymbol{\alpha})$ and $h_{2}(\boldsymbol{\alpha})$ are contained in the same orbit. Due to $\psi_{2}\left(h_{1}(\boldsymbol{\alpha})\right)=\psi_{2}\left(h_{2}(\boldsymbol{\alpha})\right)$ we have $h_{2}(\boldsymbol{\alpha})=h_{1}(\boldsymbol{\alpha})=g_{m}^{-1} h_{2}(\boldsymbol{\alpha}) g_{m}$, i.e. $g_{m} \in$ $Z\left(h_{2}(\boldsymbol{\alpha})\right)=Z$. Finally we get $g_{x}=h_{2}\left(\gamma_{x}\right)^{-1} g_{m} h_{1}\left(\gamma_{x}\right)=h_{2}\left(\gamma_{x}\right)^{-1} h_{1}\left(\gamma_{x}\right) g_{m}=g_{m}$ for all $x \in M$, i.e. $\bar{g} \in \mathbf{B}_{Z}$. Due to $h_{1}, h_{2} \in U$, we get $h_{1}=h_{2} \circ \bar{g}=h_{2}$.

4. $\Psi$ is continuous.

It is sufficient to prove that the projections from $\Psi$ onto the two factors are continuous:

- $\Psi_{1}:=\operatorname{pr}_{1} \circ \Psi$ is equal to $\pi: U \longrightarrow \pi(U)$, hence continuous.

- $\Psi_{2}:=\operatorname{pr}_{2} \circ \Psi$ is continuous as a concatenation of continuous mappings $\varphi, \psi_{2}$, $\pi_{\gamma_{x}}$ and $*$. 7

5. $\Psi$ is a homeomorphism.

Since $f$ is bijective and continuous, we only have to show (cf. Proposition G.1 in Appendix G), that every element of $\pi(U) \times \mathbf{B}_{Z} \backslash \overline{\mathcal{G}}$ has a compact neighbourhood whose preimage is again compact in $U$. Let now such an element $\left(\left[h^{\prime}\right],[\bar{g}]_{\mathbf{B}_{Z}}\right)$ be given. Since $U$ is an open subset of the compact Hausdorff space $\overline{\mathcal{A}}, U$ is locally compact. Hence, there is a compact neighbourhood $U^{\prime} \subseteq U$ of $h^{\prime}$. Now, $W:=\pi\left(U^{\prime}\right) \times \mathbf{B}_{Z} \backslash \overline{\mathcal{G}}$ is the desired compact neighbourhood of $\left(\left[h^{\prime}\right],[\bar{g}]_{\mathbf{B}_{Z}}\right):$ Due to the compactness of $U^{\prime}$ and $\overline{\mathcal{G}}, W$ is compact and by the openness of $\pi$ also a neighbourhood; moreover, $\pi^{-1}(W)=U^{\prime} \circ \overline{\mathcal{G}}$ is (again by the compactness of $\overline{\mathcal{G}}$ ) compact.

6. $\Psi$ is equivariant.

We have

$$
\begin{aligned}
\Psi(h \circ \bar{g}) & =\left([h \circ \bar{g}], \psi_{2}(\varphi(h \circ \bar{g})) *\left(\left((h \circ \bar{g})\left(\gamma_{x}\right)\right)_{x \in M}\right)\right) \\
& =\left([h], \psi_{2}\left(\varphi(h) \circ g_{m}\right) *\left(\left(g_{m}^{-1} h\left(\gamma_{x}\right) g_{x}\right)_{x \in M}\right)\right) \\
& =\left([h],\left(\psi_{2}(\varphi(h)) \cdot\left[g_{m}\right]_{Z}\right) *\left(\left(g_{m}^{-1} h\left(\gamma_{x}\right) g_{x}\right)_{x \in M}\right)\right) \\
& \quad(\cdot \text { denotes the multiplication in } Z \backslash \mathbf{G} .) \\
= & \left([h], \psi_{2}(\varphi(h)) *\left(h\left(\gamma_{x}\right) g_{x}\right)_{x \in M}\right)
\end{aligned}
$$

\footnotetext{
${ }^{4} \pi_{\gamma_{x}}: \overline{\mathcal{A}} \longrightarrow \mathbf{G}, \bar{A} \longmapsto h_{\bar{A}}\left(\gamma_{x}\right)$.

${ }^{5}$ Note that the standard theorem on the continuity of the inverse mapping is not applicable because $U$ is typically noncompact.
} 


$$
\begin{aligned}
= & \left([h],\left(\psi_{2}(\varphi(h)) *\left(h\left(\gamma_{x}\right)\right)_{x \in M}\right) \cdot[\bar{g}]_{\mathbf{B}_{Z}}\right) \\
& \quad\left(\cdot \text { now denotes multiplication in } \mathbf{B}_{Z} \backslash \overline{\mathcal{G}} .\right) \\
= & \Psi(h) \circ[\bar{g}]_{\mathbf{B}_{Z}} .
\end{aligned}
$$

qed

\subsection{Proof of Theorem 7.2}

\section{Proof Theorem 7.2}

By Proposition 2.1, $\overline{\mathcal{A}}_{\text {gen }}$ is (w.r.t. the action of $\overline{\mathcal{G}}$ ) a fibre bundle with structure group $Z \backslash \mathbf{G}$ and by Proposition 4.2 even a principal fibre bundle. The lemmata and propositions of the present section show that $\mathcal{U}$ is a covering of $\overline{\mathcal{A}}_{\text {gen }}$ by almost global trivializations.

qed

\section{Triviality of $\overline{\mathcal{A}}$ for Abelian G}

For commutative structure groups every connection is generic. Moreover, $\overline{\mathcal{A}}$ is even globally trivial:

Proposition 8.1 Let $\mathrm{G}$ be a commutative compact Lie group.

Then $\overline{\mathcal{A}}$ is a globally trivial principal fibre bundle with structure group $\overline{\mathcal{G}}_{\text {const }} \backslash \overline{\mathcal{G}}$.

Here, $\overline{\mathcal{G}}_{\text {const }}$ denoted the set of all constant gauge transforms. Obviously, $\overline{\mathcal{G}}_{\text {const }}$ equals the

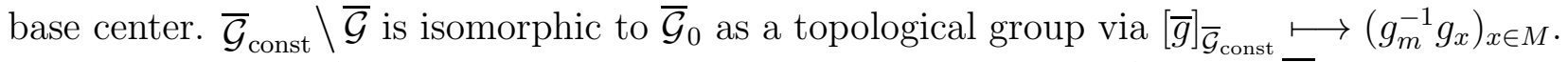
Therefore one can (after an appropriate modification of the action) regard $\overline{\mathcal{A}}$ in the abelian case as a principal $\overline{\mathcal{G}}_{0}$-fibre bundle over $\overline{\mathcal{A}} / \overline{\mathcal{G}}$.

Proof Let

$$
\begin{aligned}
\Psi: \overline{\mathcal{A}} & \longrightarrow \overline{\mathcal{A}} / \overline{\mathcal{G}} \times \overline{\mathcal{G}}_{\text {const }} \backslash \overline{\mathcal{G}}, \\
h & \longmapsto\left([h],\left[\left(h\left(\gamma_{x}\right)\right)_{x \in M}\right]\right)
\end{aligned}
$$

where $\gamma_{x}$ is as usual for all $x \in M$ some path from $m$ to $x$ being trivial for $x=m$.

In a commutative group the adjoint action is trivial, hence

$$
\overline{\mathcal{A}} / \overline{\mathcal{G}}=\operatorname{Hom}(\mathcal{H} \mathcal{G}, \mathbf{G}) / \operatorname{Ad}=\operatorname{Hom}(\mathcal{H} \mathcal{G}, \mathbf{G}) .
$$

1. $\Psi$ is surjective.

Let $[h] \in \overline{\mathcal{A}} / \overline{\mathcal{G}}$ and $[\bar{g}] \in \overline{\mathcal{G}}_{\text {const }} \backslash \overline{\mathcal{G}}$ be given. As just remarked there is an $h^{\prime} \in \overline{\mathcal{A}}$ with $\left.h^{\prime}\right|_{\mathcal{H G}}=[h]$. Now, let $h^{\prime \prime}(\gamma):=g_{x}^{-1} h^{\prime}\left(\gamma_{x} \gamma \gamma_{y}^{-1}\right) g_{y}$ for $\gamma \in \mathcal{P}_{x y}$. Obviously, $h^{\prime \prime} \in \overline{\mathcal{A}}$ and $\Psi\left(h^{\prime \prime}\right)=([h],[\bar{g}])$.

2. $\Psi$ is injective.

Let $h_{1}, h_{2} \in \overline{\mathcal{A}}$ and $\Psi\left(h_{1}\right)=\Psi\left(h_{2}\right)$. Then $\left[\left(h_{1}\left(\gamma_{x}\right)\right)_{x \in M}\right]=\left[\left(h_{2}\left(\gamma_{x}\right)\right)_{x \in M}\right]$, hence - $\gamma_{m}$ is trivial - also $h_{1}\left(\gamma_{x}\right)=h_{2}\left(\gamma_{x}\right)$ for all $x \in M$. The injectivity now follows, because two connections are equal if their holonomies are equal and if their parallel transports coincide for each $x$ along at least one path from $m$ to $x$.

3. $\Psi$ is obviously continuous.

4. $\Psi^{-1}$ is continuous because $\overline{\mathcal{A}}$ is compact and $\overline{\mathcal{A}} / \overline{\mathcal{G}} \times \overline{\mathcal{G}}_{\text {const }} \backslash \overline{\mathcal{G}}$ is Hausdorff.

5. $\Psi$ ist clearly equivariant. 


\section{Criterion for the Non-Triviality of $\overline{\mathcal{A}}_{\text {gen }}$}

We already know that $\overline{\mathcal{A}}_{\text {gen }}$ is almost globally trivial and in the abelian case even globally trivial. Now we want to know when the generic stratum is nontrivial. First we state a sufficient condition for the non-triviality of $\overline{\mathcal{A}}_{\text {gen }}$ requiring only a property of $\mathbf{G}$ and find then a class of Lie groups having this property. Finally we discuss some problems arising when we tried to prove that $\overline{\mathcal{A}}_{\text {gen }}$ is nontrivial for all non-commutative $\mathbf{G}$.

\subsection{General Criterion}

We start with the sufficient condition for the non-triviality of $\overline{\mathcal{A}}_{\text {gen }}$.

Proposition 9.1 If there is a natural number $k \geq 1$ such that $\left(\mathbf{G}^{k}\right)_{\text {gen }}$ is a nontrivial principal G-fibre bundle, then $\overline{\mathcal{A}}_{\text {gen }}$ (and thus $\overline{\mathcal{A}}$ ) is nontrivial as well.

Proof Let $k \in \mathbb{N}_{+}$and $\left(\mathbf{G}^{k}\right)_{\text {gen }}$ nontrivial.

Suppose there were a section $s: \overline{\mathcal{A}}_{\text {gen }} / \overline{\mathcal{G}} \longrightarrow \overline{\mathcal{A}}_{\text {gen }}$ for $\pi: \overline{\mathcal{A}}_{\text {gen }} \longrightarrow \overline{\mathcal{A}}_{\text {gen }} / \overline{\mathcal{G}}$. We choose a graph $\Gamma$ with $k$ edges and exactly one vertex $m$. The set of edges is denoted by $\boldsymbol{\alpha} \subseteq \mathcal{H G}$ and defines the reduction mapping $\varphi:=\varphi_{\boldsymbol{\alpha}}$. Moreover, we define $U:=\varphi^{-1}\left(\left(\mathbf{G}^{k}\right)_{\text {gen }}\right)$. Our goal is now to construct a section $s_{[\varphi]}$ in the bottom line of the diagram

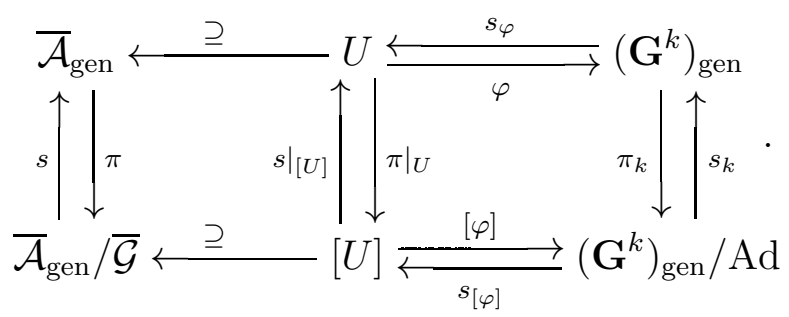

We will see that $\varphi \circ s \circ s_{[\varphi]}$ induces a continuous section for $\pi_{k}:\left(\mathbf{G}^{k}\right)_{\text {gen }} \longrightarrow$ $\left(\mathbf{G}^{k}\right)_{\text {gen }} /$ Ad that, however, does not exist by assumption.

We construct first a section $s_{\varphi}:\left(\mathbf{G}^{k}\right)_{\text {gen }} \longrightarrow \overline{\mathcal{A}}_{\text {gen }}$. Here we choose for $s_{\varphi}(\vec{g})$ that connection, that is build by means of the construction method [12] out of the trivial connection if one successively assigns the components of $\vec{g}$ to the $k$ edges in $\Gamma$. Clearly, then $\varphi\left(s_{\varphi}(\vec{g})\right)=\vec{g}$ for all $\vec{g} \in \mathbf{G}^{k}$. It is easy to see from this method, that $s_{\varphi}$ is continuous and obviously maps $\left(\mathbf{G}^{k}\right)_{\text {gen }}$ to $\overline{\mathcal{A}}_{\text {gen }}$.

Now we define for $[\vec{g}] \in\left(\mathbf{G}^{k}\right)_{\text {gen }} /$ Ad a mapping $s_{[\varphi]}:\left(\mathbf{G}^{k}\right)_{\text {gen }} / \mathrm{Ad} \longrightarrow \overline{\mathcal{A}}_{\text {gen }} / \overline{\mathcal{G}}$ by $s_{[\varphi]}([\vec{g}]):=\pi\left(s_{\varphi}(\vec{g})\right)$.

- $s_{[\varphi]}$ is well-defined.

Let $\vec{g}_{2}=\vec{g}_{1} \circ g, g \in \mathbf{G}$. Then $s_{\varphi}\left(\vec{g}_{2}\right)=s_{\varphi}\left(\vec{g}_{1}\right) \circ \bar{g}$, where $\bar{g} \in \overline{\mathcal{G}}$ is that gauge transform having the value $g$ everywhere. Hence $s_{[\varphi]}\left(\left[\vec{g}_{1}\right]\right)=s_{[\varphi]}\left(\left[\vec{g}_{2}\right]\right)$.

- $s_{[\varphi]}$ is a section.

As just proven we have $s_{[\varphi]} \circ \pi_{k}=\pi \circ s_{\varphi}$, thus

\footnotetext{
${ }^{6}$ Let $e$ be some edge in $M$. There are at most two indices $i$ and $j$ such that the initial paths of $\alpha_{i}$ and $\alpha_{j}$ coincide with a partial path of $e$ or $e^{-1}$. If there were no such indices, then $\pi_{e} \circ s_{\varphi}(\vec{g})=e_{\mathbf{G}}$ for all $\vec{g}$. Otherwise $\pi_{e} \circ s_{\varphi}(\vec{g})$ equals a product of $g_{i}, g_{j}, g_{i}^{-1}$ or $g_{j}^{-1}$. Thus, in any case $\pi_{e} \circ s_{\varphi}: \mathbf{G}^{k} \longrightarrow \mathbf{G}$ is continuous for all edges $e$. Hence, $s_{\varphi}$ is continuous as well.
} 


$$
\begin{array}{rlr}
{[\varphi] \circ s_{[\varphi]}} & =[\varphi] \circ s_{[\varphi]} \circ \pi_{k} \circ\left(\pi_{k}\right)^{-1} & \text { (Surjectivity of } \pi_{k} \text { ) } \\
& =[\varphi] \circ \pi \circ s_{\varphi} \circ\left(\pi_{k}\right)^{-1} & \\
& =\pi_{k} \circ \varphi \circ s_{\varphi} \circ\left(\pi_{k}\right)^{-1} & \text { (Commutativity of projections) } \\
& =\pi_{k} \circ\left(\pi_{k}\right)^{-1} & \text { (Section property) } \\
& =\mathrm{id}_{\left(\mathbf{G}^{k}\right)_{\operatorname{gen}} / \mathrm{Ad} .} & \text { (Surjectivity of } \pi_{k} \text { ) }
\end{array}
$$

- $s_{[\varphi]}$ is continuous.

By the quotient criterion, $s_{[\varphi]}$ is continuous iff $s_{[\varphi]} \circ \pi_{k}$ is continuous. But, the latter one is equal to $\pi \circ s_{\varphi}$, hence continuous.

Finally we prove that $s_{k}:=\varphi \circ s \circ s_{[\varphi]}$ is a section for $\pi_{k}$ : We have

$$
\pi_{k} \circ s_{k}=\pi_{k} \circ \varphi \circ s \circ s_{[\varphi]}=[\varphi] \circ \pi \circ s \circ s_{[\varphi]}=\operatorname{id}_{\left(\mathbf{G}^{k}\right)_{\operatorname{gen}} / \mathrm{Ad}}
$$

because $s$ and $s_{[\varphi]}$ are sections themselves. The continuity of $s_{k}$ is clear. Hence, there is a global continuous section in $\pi_{k}:\left(\mathbf{G}^{k}\right)_{\text {gen }} \longrightarrow\left(\mathbf{G}^{k}\right)_{\text {gen }} /$ Ad. This is a contradiction to the assumption that $\pi_{k}$ is a nontrivial bundle.

Therefore, there is no continuous section over the whole $\overline{\mathcal{A}}_{\text {gen }} / \overline{\mathcal{G}}$ qed

\subsection{Concrete Criterion}

The crucial question is now what concrete $\mathbf{G}$ and $k$ give nontrivial bundles $\pi_{k}:\left(\mathbf{G}^{k}\right)_{\text {gen }} \longrightarrow$ $\left(\mathbf{G}^{k}\right)_{\text {gen }} / \mathrm{Ad}$. It is quite easy to see (cf. Appendix $\left.\mathbf{B}\right)$ that in the case of $\mathbf{G}=S U(2)$ the bundle is empty for $k=1$, trivial for $k=2$ and nontrivial for $k \geq 3$. So maybe typically up to some $k$ the bundles are trivial, but nontrivial for bigger $k$. But, is there a $k$ for every non-abelian $\mathrm{G}$ such that $\pi_{k}$ is nontrivial?

Up to now, we did not find a complete answer. However, the following two propositions give a wide class of groups, for which the bundle is nontrivial starting at some $k$. In particular, the proposition above is non-empty, i.e., its assumptions can be fulfilled.

Proposition 9.2 Let $\mathbf{G}$ be a non-abelian Lie group with $\pi_{1}^{\mathrm{h}}(Z \backslash \mathbf{G}) \neq 1$ and $\pi_{1}^{\mathrm{h}}(\mathbf{G})=1$.ป Then there is a $k \in \mathbb{N}$ such that $\left(\mathbf{G}^{k}\right)_{\text {gen }}$ is a nontrivial principal G-fibre bundle.

Proof - Choose $k^{\prime} \in \mathbb{N}$ so large that $\left(\mathrm{G}^{k^{\prime}}\right)_{\text {gen }}$ is non-empty (cf. Proposition 5.2).

By general arguments one sees [6] that the codimension of all non-generic strata, i.e. all strata whose type is smaller than $[Z]$, is at least 1 .

By Corollary E.2 in Appendix $\mathbb{E}$ the non-generic strata in $\mathbf{G}^{3 k^{\prime}}$ have at least codimension 3. Let $k:=3 k^{\prime}$.

- Suppose, the principal fibre bundle $\left(\mathbf{G}^{k}\right)_{\text {gen }}$ were trivial.

Then $\left(\mathbf{G}^{k}\right)_{\text {gen }} \cong\left(\mathbf{G}^{k}\right)_{\text {gen }} / \mathrm{Ad} \times Z \backslash \mathbf{G}$, hence in particular

$$
\pi_{1}^{\mathrm{h}}\left(\left(\mathbf{G}^{k}\right)_{\text {gen }}\right) \cong \pi_{1}^{\mathrm{h}}\left(\left(\mathbf{G}^{k}\right)_{\text {gen }} / \mathrm{Ad}\right) \oplus \pi_{1}^{\mathrm{h}}(Z \backslash \mathbf{G}) .
$$

Since because of the compactness of $\mathbf{G}$ the number of non-generic strata in $\mathbf{G}^{k}$ is finite [26] and each one of the strata is a submanifold of $\mathbf{G}^{k}$ [6] having codimension bigger or equal 3, we have $\pi_{1}^{\mathrm{h}}\left(\left(\mathbf{G}^{k}\right)_{\text {gen }}\right) \cong \pi_{1}^{\mathrm{h}}\left(\mathbf{G}^{k}\right)$. Consequently, (1) reduces to

$$
\pi_{1}^{\mathrm{h}}(\mathbf{G})^{k} \cong \pi_{1}^{\mathrm{h}}\left(\mathbf{G}^{k}\right) \cong \pi_{1}^{\mathrm{h}}\left(\left(\mathbf{G}^{k}\right)_{\text {gen }} / \mathrm{Ad}\right) \oplus \pi_{1}^{\mathrm{h}}(Z \backslash \mathbf{G})
$$

This, however, is contradiction to the assumptions $\pi_{1}^{\mathrm{h}}(\mathbf{G})=1$ and $\pi_{1}^{\mathrm{h}}(Z \backslash \mathbf{G}) \neq 1$. Hence, $\left(\mathbf{G}^{k}\right)_{\text {gen }}$ is nontrivial.

qed

\footnotetext{
${ }^{7}$ We denote the fundamental group not as usual by $\pi_{1}$, but by $\pi_{1}^{\mathrm{h}}$, in order to avoid confusion with $\pi_{k}: \mathbf{G}^{k} \longrightarrow \mathbf{G}^{k} /$ Ad.
} 
Proposition 9.3 The assumptions of the proposition above are fulfilled, in particular, for all semisimple (simply connected) Lie groups whose decomposition into simple Lie groups contains at least one of the factors $A_{n}, B_{n}, C_{n}, D_{n}, E_{6}$ or $E_{7}$.

Proof It is well-known that among the simple (and simply connected) compact Lie groups exactly the representatives of the series listed above have nontrivial center. [16]

The assumption now follows, because for simply connected $\mathbf{G}$ the order of $Z$ equals $\pi_{1}^{\mathrm{h}}(Z \backslash \mathbf{G})$ [17] and the center of the direct product of groups equals the direct product of the corresponding centers.

qed

In particular, we see that $\overline{\mathcal{A}}_{\text {gen }}$ is nontrivial for all $\mathbf{G}=S U(N)\left(=A_{N-1}, N \geq 2\right)$. However, the corresponding problem, e.g., for $\mathbf{G}=S O(N)$ or the prominent case $\mathbf{G}=E_{8} \times E_{8}$ remains unsolved.

We remark that in general for fixed $\mathbf{G}$ the bundles gets "more nontrivial" when $k$ increases. Strictly speaking, we have

Proposition 9.4 For every $\mathbf{G}$ the non-triviality of $\left(\mathbf{G}^{k}\right)_{\text {gen }}$ implies that of $\left(\mathbf{G}^{k+1}\right)_{\text {gen }}$.

Proof Let $k$ be chosen such that $\left(\mathbf{G}^{k}\right)_{\text {gen }}$ is nontrivial. Suppose there is a section $s_{k+1}$ for $\pi_{k+1}:\left(\mathbf{G}^{k+1}\right)_{\text {gen }} \longrightarrow\left(\mathbf{G}^{k+1}\right)_{\text {gen }} / \mathrm{Ad}$.

We get the following commutative diagram

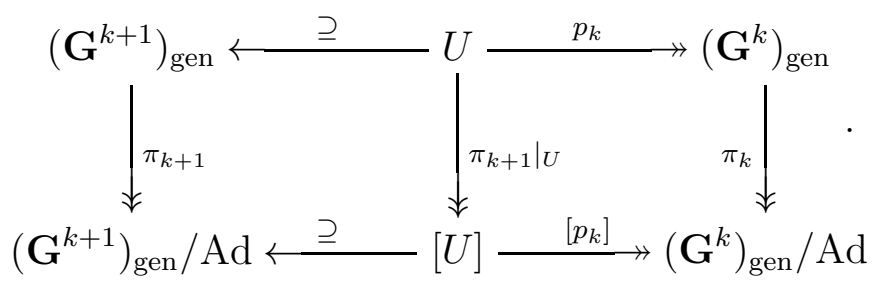

Here $p_{k}:\left(\mathbf{G}^{k+1}\right)_{\text {gen }} \longrightarrow\left(\mathbf{G}^{k}\right)_{\text {gen }}$ is the projection onto the first $k$ coordinates and $\left[p_{k}\right]$ is induced in a natural way. Additionally, we defined $U:=p_{k}^{-1}\left(\left(\mathbf{G}^{k}\right)_{\text {gen }}\right)$.

Now we reuse the idea for the proof of Proposition 9.1. First we set $i_{k}:\left(\mathbf{G}^{k}\right)_{\text {gen }} \longrightarrow$ $U=\left(\mathbf{G}^{k}\right)_{\text {gen }} \times \mathbf{G}$ with $i_{k}(\vec{g}):=\left(\vec{g}, e_{\mathbf{G}}\right)$, where the rhs vector is viewed as an element of $\mathbf{G}^{k+1}$. Obviously, $i_{k}$ is a continuous section for $p_{k}$, that additionally - as can be checked quickly - defines a continuous section for $\left[p_{k}\right]$ via $\left[i_{k}\right]([\vec{g}]):=\pi_{k+1}\left(i_{k}(\vec{g})\right)$. Finally one sees that $s_{k}:=\left.p_{k} \circ s_{k+1}\right|_{[U]} \circ\left[i_{k}\right]$ is a section for $\pi_{k}$. This, however, is a contradiction to the non-triviality of $\pi_{k}$.

qed

\subsection{Conjecture}

Let us return again to the proof of Proposition 9.1. There we deduced via $\varphi$ from the nontriviality of the generic stratum in $\mathbf{G}^{k}$ that the preimage $\varphi^{-1}\left(\left(\mathbf{G}^{k}\right)_{\text {gen }}\right)$, hence $\overline{\mathcal{A}}_{\text {gen }}$ as well is a nontrivial bundle. But, besides we know that $\varphi$ is surjective even as a mapping from $\overline{\mathcal{A}}_{\text {gen }}$ to the whole $\mathrm{G}^{k}$. 13. It seems to be obvious that one can now deduce from the non-existence of a section for $\pi_{k}$ over the whole space $\mathbf{G}^{k} / \mathrm{Ad}$ (and not only over the generic stratum as above) analogously to the case above the non-existence of a global section in $\overline{\mathcal{A}}_{\text {gen }} \longrightarrow \overline{\mathcal{A}}_{\text {gen }} / \overline{\mathcal{G}}$. But, the existence of a section for the whole $\pi_{k}$ is rather not to be expected because typically in the case of non-commutative structure groups $\mathbf{G}$ the mapping $\pi_{k}$ does not define a fibre 
bundle. (This can easily be seen because in $\mathbf{G}^{k}$ there occurs both the orbit [i.e. the fibre] $Z \backslash \mathbf{G}$ and the orbit pt $=\mathbf{G} \backslash \mathbf{G}$ being never isomorphic. However, this is not a criterion for the non-existence of a section, but simply just an indication.) Hence, one can guess that $\overline{\mathcal{A}}_{\text {gen }}$ is surely nontrivial for non-commutative $\mathbf{G}$, at least as far as $\pi_{k}$ possesses no section over the whole $\mathrm{G}^{k} / \mathrm{Ad}$.

Unfortunately, we were not able to prove this up to now. At one point the proof above uses explicitly the fact that only the generic stratum in $\mathbf{G}^{k}$ is considered - namely, for the definition of $s_{[\varphi]}$. A continuation of that mapping from the generic elements of $\left(\mathbf{G}^{k}\right)_{\operatorname{gen}} / \mathrm{Ad}$ to the whole $\mathbf{G}^{k} / \mathrm{Ad}$ is not possible as the next proposition shows:

Proposition 9.5 Let $k \in \mathbb{N}_{+}$be some number for that there are both generic and nongeneric elements in $\mathbf{G}^{k}$.

Then there is no continuous mapping $s_{\varphi}: \mathbf{G}^{k} \longrightarrow \overline{\mathcal{A}}_{\text {gen }}$, such that $s_{[\varphi]}$ : $\mathrm{G}^{k} / \mathrm{Ad} \longrightarrow \overline{\mathcal{A}}_{\text {gen }} / \overline{\mathcal{G}}$ with $s_{[\varphi]}([\vec{g}]):=\pi\left(s_{\varphi}(\vec{g})\right)$ is well-defined and that $\varphi \circ s_{\varphi}=\operatorname{id}_{\mathbf{G}^{k}}$.

We need the following

Lemma 9.6 Let $k, l \in \mathbb{N}_{+}$such that there are generic elements in $\mathbf{G}^{l}$.

Then we have: $\mathbf{G}$ is abelian iff there is a continuous $f: \mathbf{G}^{l} \longrightarrow \mathbf{G}^{k}$ with

- $\vec{g}_{1} \sim \vec{g}_{2} \Longrightarrow\left(\vec{g}_{1}, f\left(\vec{g}_{1}\right)\right) \sim\left(\vec{g}_{2}, f\left(\vec{g}_{2}\right)\right)$ and

- $Z(\vec{g}) \cap Z(f(\vec{g}))=Z$ for all $\vec{g} \in \mathbf{G}^{l}$.

Proof - Let $\mathbf{G}$ be abelian. Then, e.g., $f(\vec{g})=e_{\mathbf{G}}, \vec{g} \in \mathbf{G}^{l}$, fulfills the conditions of the lemma.

- Let $\mathbf{G}$ be non-abelian. Suppose there were such an $f$. Let $\vec{g}_{1}, \vec{g}_{2} \in \mathbf{G}^{l}$ be equivalent, i.e., let there exist a $g \in \mathbf{G}$ with $\vec{g}_{2}=\vec{g}_{1} \circ g$. By assumption there is also a $g^{\prime} \in \mathbf{G}$ with $\left(\vec{g}_{2}, f\left(\vec{g}_{2}\right)\right)=\left(\vec{g}_{1}, f\left(\vec{g}_{1}\right)\right) \circ g^{\prime}=\left(\vec{g}_{1} \circ g^{\prime}, f\left(\vec{g}_{1}\right) \circ g^{\prime}\right)$. Hence, $\vec{g}_{1} \circ g=\vec{g}_{1} \circ g^{\prime}$, i.e. $g^{\prime}=g^{\prime \prime} g$ for some $g^{\prime \prime} \in Z\left(\vec{g}_{1}\right)$. Consequently, $f\left(\vec{g}_{1} \circ g\right)=$ $f\left(\vec{g}_{2}\right)=f\left(\vec{g}_{1}\right) \circ g^{\prime}=f\left(\vec{g}_{1}\right) \circ g^{\prime \prime} \circ g$. In particular, for all generic $\vec{g}_{1}$ we have $g^{\prime \prime} \in Z$, i.e. $f\left(\vec{g}_{1} \circ g\right)=f\left(\vec{g}_{1}\right) \circ g$. Since the generic elements by assumption form a dense subset in $\mathbf{G}^{l}$ (cf. Proposition 5.1) and $f$ is to be continuous, $f\left(\vec{g}_{1} \circ g\right)=f\left(\vec{g}_{1}\right) \circ g$ has to hold even for all $\vec{g}_{1} \in \mathbf{G}^{l}$ and all $g \in \mathbf{G}$. Let $\vec{g}$ now be a non-generic element in $\mathbf{G}^{l}$, i.e., let there exist a $g \in Z(\vec{g}) \backslash Z$. But, now $f(\vec{g})=f(\vec{g} \circ g)=f(\vec{g}) \circ g$, hence $g \in Z(f(\vec{g})) \cap(Z(\vec{g}) \backslash Z)=(Z(\vec{g}) \cap Z(f(\vec{g}))) \backslash Z=\varnothing$. Therefore all $\vec{g} \in \mathbf{G}^{l}$ are generic in contradiction to the non-commutativity of $\mathbf{G}$.

qed

\section{Proof Proposition 9.5}

Suppose there exists such an $s_{\varphi}: \mathrm{G}^{k} \longrightarrow \overline{\mathcal{A}}_{\text {gen }}$.

1. Let $\vec{g} \in \mathbf{G}^{k}$ be arbitrary, but fixed. Due to $s_{\varphi}(\vec{g}) \in \overline{\mathcal{A}}_{\text {gen }}$ there is an $\boldsymbol{\alpha}_{\vec{g}} \subseteq \mathcal{H} \mathcal{G}$ with $h_{s_{\varphi}(\vec{g})}\left(\boldsymbol{\alpha}_{\vec{g}}\right) \in\left(\mathbf{G}^{\# \boldsymbol{\alpha}_{\vec{g}}}\right)_{\text {gen }}$. Since the generic stratum is always open and since together with $s_{\varphi}$ and $h_{\boldsymbol{\alpha}_{\vec{g}}}$ also $h_{\boldsymbol{\alpha}_{\vec{g}}} \circ s_{\varphi}: \mathbf{G}^{k} \longrightarrow \mathbf{G}^{\# \boldsymbol{\alpha}_{\vec{g}}}$ is continuous, $U_{\vec{g}}:=\left(h_{\boldsymbol{\alpha}_{\vec{g}}} \circ s_{\varphi}\right)^{-1}\left(\left(\mathbf{G}^{\# \boldsymbol{\alpha}_{\vec{g}}}\right)_{\text {gen }}\right)$ defines an open neighbourhood of $\vec{g}$.

2. Varying over all $\vec{g}$ one gets an open covering $\mathcal{U}:=\left\{U_{\vec{g}} \mid \vec{g} \in \mathbf{G}^{k}\right\}$ of $\mathbf{G}^{k}$. Since $\mathbf{G}$ is compact, there are finitely many $\vec{g}_{i} \in \mathbf{G}^{k}$ such that $\bigcup_{i} U_{\vec{g}_{i}}=\mathbf{G}^{k}$. Let now $\boldsymbol{\alpha}^{\prime}$ be the set (the tuple, respectively) of all these $\boldsymbol{\alpha}_{\vec{g}_{i}}$.

3. We define $f:=h_{\boldsymbol{\alpha}^{\prime}} \circ s_{\varphi}: \mathbf{G}^{k} \longrightarrow \mathbf{G}^{\# \boldsymbol{\alpha}^{\prime}}$ and - recall $\varphi \equiv h_{\boldsymbol{\alpha}}-$

$$
f^{\prime}:=\left(h_{\boldsymbol{\alpha}} \circ s_{\varphi}, h_{\boldsymbol{\alpha}^{\prime}} \circ s_{\varphi}\right) \equiv\left(\mathrm{id}_{\mathbf{G}^{k}}, f\right): \mathbf{G}^{k} \longrightarrow \mathbf{G}^{k+\# \boldsymbol{\alpha}^{\prime}} \text {. }
$$

We have: 
- $f$ is continuous.

- Let $\vec{g}^{\prime}, \vec{g}^{\prime \prime} \in \mathbf{G}^{k}$ with $\vec{g}^{\prime} \sim \vec{g}^{\prime \prime}$. From that, due to the assumed well-definedness of $s_{[\varphi]}, s_{\varphi}\left(\vec{g}^{\prime}\right) \sim s_{\varphi}\left(\vec{g}^{\prime \prime}\right)$ w.r.t. $\overline{\mathcal{G}}$. Hence, in particular $\left(\vec{g}^{\prime}, f\left(\vec{g}^{\prime}\right)\right)=f^{\prime}\left(\vec{g}^{\prime}\right) \sim$ $f^{\prime}\left(\vec{g}^{\prime \prime}\right)=\left(\vec{g}^{\prime \prime}, f\left(\vec{g}^{\prime \prime}\right)\right)$.

- $Z(f(\vec{g}))=Z$ for all $\vec{g} \in \mathbf{G}^{k}$.

Let $\vec{g} \in \mathbf{G}^{k}$. Then there is an $i$ with $\vec{g} \in U_{\vec{g}_{i}}$. Thus, $h_{\boldsymbol{\alpha}_{\vec{g}_{i}}}\left(s_{\varphi}(\vec{g})\right) \in\left(\mathbf{G}^{\# \boldsymbol{\alpha}_{\vec{g}_{i}}}\right)_{\text {gen }}$, hence $f(\vec{g})=h_{\boldsymbol{\alpha}^{\prime}} \circ s_{\varphi}(\vec{g}) \in\left(\mathbf{G}^{\# \boldsymbol{\alpha}^{\prime}}\right)_{\text {gen }}$ due to $\boldsymbol{\alpha}_{\vec{g}_{i}} \subseteq \boldsymbol{\alpha}^{\prime}$.

Due to $Z \subseteq Z(\vec{g}) \cap Z(f(\vec{g})) \subseteq Z(f(\vec{g}))=Z$, $f$ fulfills all assumptions of the preceding lemma, i.e., $\mathbf{G}$ is abelian in contradiction to the supposition. $\quad$ qed

Despite these obstacles we close this section with an even stronger

Conjecture $\mathbf{9 . 7} \overline{\mathcal{A}}_{\text {gen }}$ is nontrivial for every non-commutative $\mathbf{G}$.

Perhaps, there is even for every non-commutative $\mathbf{G}$ a $k$ such that $\left(\mathbf{G}^{k}\right)_{\text {gen }}$ is nontrivial.

\section{Discussion}

We summarize the results of this paper on the structure of the generic stratum of $\overline{\mathcal{A}}$, noting that the assertions of the following theorem are completely independent of the base manifold $M$ :

Theorem 10.1 1. $\overline{\mathcal{A}}_{\text {gen }}$ has the induced Haar measure 1.

2. $\overline{\mathcal{A}}_{\text {gen }}$ is a $\mu_{0}$-almost globally trivial principal $\overline{\mathcal{G}}$-fibre bundle with structure group $\mathbf{B}_{Z} \backslash \overline{\mathcal{G}}$.

3. $\overline{\mathcal{A}}_{\text {gen }}$ is globally trivial for abelian $\mathbf{G}$.

4. $\overline{\mathcal{A}}_{\text {gen }}$ is not globally trivial for nonabelian, simply connected $\mathrm{G}$ with nontrivial center.

Here $\mathbf{B}_{Z} \subseteq \overline{\mathcal{G}}$ is the set of all constant, center-valued gauge transforms.

Hence, the Gribov problem - well-known in the case of regular (Sobolev) connections for a long time - appears in the Ashtekar approach as well. However, we could refine the statement substantially: The non-triviality is now concentrated on a zero subset. Just this fact has large physical importance - it justifies the definition of the induced Haar measure on $\overline{\mathcal{A}} / \overline{\mathcal{G}}$ by the image measure of the corresponding measure on $\overline{\mathcal{A}}$. Actually, there are two possibilities for the choice of such a measure. $]^{0}$

Let $X$ be some topological space equipped with a measure $\mu$ and let $G$ be some topological group acting on $X$. What should the corresponding measure $\mu_{G}$ on the orbit space $X / G$ look like? On the one hand, one could simply define $\mu_{G, 1}(U):=\pi_{*} \mu(U) \equiv \mu\left(\pi^{-1}(U)\right)$ for all measurable $U \subseteq X / G$, i.e. use the image measure w.r.t. the canonical projection $\pi: X \longrightarrow$ $X / G$. On the other hand, one could perform a kind of Faddeev-Popov transformation: Let us assume that $X$ can be written as a disjoint union of certain sets $V$ and that each $V$ equals $V / G \times G_{V} \backslash G$ where $G_{V}$ characterizes the type of orbits on $V$. Then one could define the measure of a set $U$ that w.l.o.g. is contained in one of these $V / G$ naively by

$$
\mu_{G, 2}(U):=\mu\left(\pi^{-1}(U)\right) \nu\left(G_{V}\right)
$$

\footnotetext{
${ }^{8}$ For an earlier discussion see [13].
} 
where $\nu$ somehow measures the "size" of the stabilizer $G_{V}$ in $G$. (The measure of a general $U$ can then be defined via $\mu_{G, 2}(U):=\sum_{V} \mu_{G, 2}(U \cap(V / G))$.) In contrast to the first method, here the orbit space and not the total space is regarded to be primary: If the measure is uniformly distributed over all points of the total space, the image measure on the orbits would in the first case no longer be uniformly distributed; the weighting of the orbits comes along according to their size. But, in the second case the uniform distribution remains, i.e. the group degrees of freedom do not play any rôle in Faddeev-Popov context. That the equation (2) above can indeed be seen as a Faddeev-Popov transformation will be clear by means of a small rearrangement. Namely, we have'?

hence

$$
\mu\left(\pi^{-1}(U)\right)=\frac{1}{\nu\left(G_{V}\right)} \mu_{G, 2}(U)
$$

$$
\begin{aligned}
\pi_{*} \mu=\Delta \odot \mu_{G, 2} \quad \text { with } \quad \Delta: X & \longrightarrow \frac{\widehat{\mathbb{R}}}{\nu} \\
x & \longmapsto \frac{1}{\nu(\operatorname{Stab}(x))}
\end{aligned}
$$

Thus, $\frac{1}{\nu\left(G_{V}\right)}=\Delta$ is nothing but the Faddeev-Popov determinant.

But, what does this mean for our concrete problem $X=\overline{\mathcal{A}}, G=\overline{\mathcal{G}}$ and $\mu=\mu_{0}$ ? Since the stabilizer of a connection in the generic stratum is minimal, $\Delta$ is maximal on $\overline{\mathcal{A}}_{\text {gen }}$. Moreover, $\Delta$ has to be constant on the whole $\overline{\mathcal{A}}_{\text {gen }}$. Since we can assume that $\Delta$ is not identically 0 , we get the measure $\mu_{0,2}$ on $\overline{\mathcal{A}}_{\text {gen }} / \overline{\mathcal{G}}$ directly from (3). Hence, we are left with the nongeneric strata only. We know already that the stabilizer of a connection is isomorphic to the corresponding holonomy centralizer. But, typically this subgroup of $\mathbf{G}$ has a higher dimension than the center has, i.e. its measure w.r.t. $\nu$ should be infinite. Hence, $\Delta$ would vanish on the nongeneric connections, and a complete determination of $\mu_{0,2}$ via (3) is no longer possible. The simplest way out would be of course to give the nongeneric strata simply the $\mu_{0,2}$-measure 0 . This is plausible, if one takes into account that, on the one hand, in the case of (sufficiently smooth) actions of Lie groups on manifolds the nonmaximal strata have lower dimension than the maximal stratum has even after being projected down to the orbit space, i.e. they can be regarded as zero subsets, and, on the other hand, the measure on $\overline{\mathcal{A}} / \overline{\mathcal{G}}$ could be defined using the projections $\pi_{v}: \overline{\mathcal{A}} / \overline{\mathcal{G}} \longrightarrow \mathrm{G}^{k} /$ Ad. Nevertheless, it remains for the time being unclear, whether one excludes this way perhaps physically interesting phenomena. However, the danger is relatively small. The measure $\mu_{0,2}$ is, of course, not yet the "dynamical" measure, but simply a kind of "kinematical" measure. This serves only as a - in a certain sense natural - background measure by that means using the dynamics of the system the "physical" measure can be constructed. One could even argue that this kinematical measure should contain no dynamical information at all. Using this point of view - and using the arguments above on the measures of nongeneric strata - we think it is meaningful to give these nongeneric strata in $\overline{\mathcal{A}} / \overline{\mathcal{G}}$ the induced Haar measure 0 .

Therewith we realize that both possibilities above for defining a measure on the orbit space are equivalent in the case of $\overline{\mathcal{A}} / \overline{\mathcal{G}}$. Here $\Delta$ is constant up to a zero subset, hence identical 1 , i.e., we have $\mu_{0,1}=\pi_{*} \mu_{0}=\Delta \odot \mu_{0,2}=\mu_{0,2}$. Additionally, we see that we can restrict ourselves to an almost global trivialization of $\overline{\mathcal{A}}_{\text {gen }}$ when integrating. Consequently, the Gribov problem appearing for certain $\mathbf{G}$ is irrelevant, e.g., for the calculation of expectation values w.r.t. $\mu_{0}$. Moreover: This statement is true even for all measures that are absolutely continuous w.r.t.

\footnotetext{
${ }^{9}$ We drop - in contrast to the Faddeev-Popov strategy in the introduction - the (fixed) measure $\nu^{\prime}(G)$ of the totally acting group.
} 
$\mu_{0}$. Therefore, the next step in this context should be an investigation of the structure of physically interesting measures.

\section{Acknowledgements}

The author has been supported in part by the Reimar-Lüst-Stipendium of the Max-PlanckGesellschaft. The idea of using homotopy arguments for the proof of the non-triviality on the Lie group level is due to Lorenz Schwachhöfer. Moreover, the author thanks Detlev Buchholz, Hartmann Römer, Gerd Rudolph and Matthias Schmidt for inspiring discussions.

\section{Appendix}

\section{A Group Isomorphy of the Two Normalizers}

In Section 2 we dealt with structure groups of the strata. As in the case of the stabilizer of a connection we could describe each of these groups using by far simpler structures in the underlying Lie group $\mathbf{G}$. We saw that

$$
\mathbf{B}(\bar{A}) \backslash N(\mathbf{B}(\bar{A})) \text { and } Z\left(\mathbf{H}_{\bar{A}}\right) \backslash N\left(Z\left(\mathbf{H}_{\bar{A}}\right)\right) \times \underset{x \neq m}{\times} Z\left(Z\left(\mathbf{H}_{\bar{A}}\right)\right)
$$

are homeomorphic. In this appendix we are going to discuss whether these groups are isomorphic even as topological groups.

In order to reduce the size of the expressions in what follows, we assume w.l.o.g. that the connection $\bar{A} \in \overline{\mathcal{A}}$ being under consideration has the property that $h_{\bar{A}}\left(\gamma_{x}\right)=e_{\mathbf{G}}$ for all $x \in M$ where $\gamma_{x}$ is as usual some fixed path from $m$ to $x$ and $\gamma_{m}$ is trivial. This, indeed, is no restriction because every $\bar{A}^{\prime} \in \overline{\mathcal{A}}$ is gauge equivalent to such an $\bar{A}$. For this, one would simply set $\bar{g}:=\left(h_{\bar{A}^{\prime}}\left(\gamma_{x}\right)^{-1}\right)_{x \in M} \in \overline{\mathcal{G}}$ and $\bar{A}:=\bar{A}^{\prime} \circ \bar{g}$.

As we noticed in the main text already, we will restrict ourselves to so-called "reasonable" isomorphisms.

1. We look only for isomorphisms between

$$
\mathbf{B}(\bar{A}) \backslash N(\mathbf{B}(\bar{A})) \text { and } Z\left(\mathbf{H}_{\bar{A}}\right) \backslash N\left(Z\left(\mathbf{H}_{\bar{A}}\right)\right) \times \underset{x \neq m}{\times} Z\left(Z\left(\mathbf{H}_{\bar{A}}\right)\right)
$$

that are induced by an isomorphism

$$
\psi: N(\mathbf{B}(\bar{A})) \longrightarrow N\left(Z\left(\mathbf{H}_{\bar{A}}\right)\right) \times \underset{x \neq m}{\times} Z\left(Z\left(\mathbf{H}_{\bar{A}}\right)\right)
$$

between the two non-factorized spaces. This means, such a factor isomorphism has to be a continuation of the natural isomorphism between the base centralizer and the holonomy centralizer, hence fulfill $\psi(\mathbf{B}(\bar{A}))=Z\left(\mathbf{H}_{\bar{A}}\right) \times \mathbf{X}_{x \neq m}\left\{e_{\mathbf{G}}\right\}$.

2. We denote the mapping, that one gets by concatenation of $\psi$ and of the corresponding projection to the $x$-component of the image space, by $\psi_{x}, x \in M$. For a "reasonable" isomorphism $\psi$ we demand

a) $\psi_{m} \equiv \pi_{m}: N(\mathbf{B}(\bar{A})) \longrightarrow N\left(Z\left(\mathbf{H}_{\bar{A}}\right)\right)$ and

b) $\psi_{x}(\bar{g})$ depends only on the values of $\bar{g}$ in $x$ and $m$.

We think viewing at Proposition 2.2 these restrictions are natural. We neglect only "wild" isomorphisms, i.e. mappings that mix the points of $M$.

Now, let $\psi$ be a "reasonable" isomorphism. We fix a point $x \neq m$ and investigate, how the projection $\psi_{x}$ of $\psi$ to the point $x$ has to look like. 
Since $\psi$ is to be a "reasonable" homomorphism, $\psi_{x}$ is a map from $\left(g_{m}, g_{x}\right)$ to $\psi_{x}(\bar{g}) \equiv$ $\psi_{x}\left(g_{m}, g_{x}\right)$. By Proposition 2.2 every $\bar{g} \in N(\mathbf{B}(\bar{A}))$ is just determined by the values of $g_{m} \in N\left(Z\left(\mathbf{H}_{\bar{A}}\right)\right)$ and of $z_{x} \in Z\left(Z\left(\mathbf{H}_{\bar{A}}\right)\right)$ : we have $g_{x}=z_{x} g_{m}$. Hence, $\psi_{x}$ is well-defined ifftण

$$
\psi_{x}(g, z g) \in Z\left(Z\left(\mathbf{H}_{\bar{A}}\right)\right) \text { for all } g \in N\left(Z\left(\mathbf{H}_{\bar{A}}\right)\right) \text { and } z \in Z\left(Z\left(\mathbf{H}_{\bar{A}}\right)\right) \text {. }
$$

The homomorphy property implies $\psi\left(\bar{g}_{1}\right) \psi\left(\bar{g}_{2}\right)=\psi\left(\bar{g}_{1} \bar{g}_{2}\right)$ for all $\bar{g}_{i} \in N(\mathbf{B}(\bar{A}))$, hence

$$
\psi_{x}\left(g_{1} g_{2}, z_{1} g_{1} z_{2} g_{2}\right)=\psi_{x}\left(g_{1}, z_{1} g_{1}\right) \psi_{x}\left(g_{2}, z_{2} g_{2}\right) \text { for all } g_{i} \in N\left(Z\left(\mathbf{H}_{\bar{A}}\right)\right) \text { and } z_{i} \in Z\left(Z\left(\mathbf{H}_{\bar{A}}\right)\right) \text {. }
$$

Now, we define

and

$$
\chi(z):=\psi_{x}(1, z) \text { for } z \in Z\left(Z\left(\mathbf{H}_{\bar{A}}\right)\right)
$$

$$
\varphi(g):=\psi_{x}(g, g) \text { for } g \in N\left(Z\left(\mathbf{H}_{\bar{A}}\right)\right)
$$

Obviously $\chi: Z\left(Z\left(\mathbf{H}_{\bar{A}}\right)\right) \longrightarrow Z\left(Z\left(\mathbf{H}_{\bar{A}}\right)\right)$ and $\varphi: N\left(Z\left(\mathbf{H}_{\bar{A}}\right)\right) \longrightarrow Z\left(Z\left(\mathbf{H}_{\bar{A}}\right)\right)$ are continuous (hence smooth) homomorphisms and we have

$$
\psi_{x}(g, z g)=\chi(z) \varphi(g)
$$

$\chi$ is even an automorphism of $Z\left(Z\left(\mathbf{H}_{\bar{A}}\right)\right)$ because $\chi$ is per constr. an injective Lie morphism. $\square$ The injectivity of $\chi$ here is a consequence of our assumption that $\psi_{m}$ is trivial and $\psi_{x}(\bar{g})$ does not depend on $g_{x^{\prime}}, x^{\prime} \neq x, m$.

Now let $g \in N\left(Z\left(\mathbf{H}_{\bar{A}}\right)\right)$ with $\varphi(g) \in Z\left(Z\left(Z\left(\mathbf{H}_{\bar{A}}\right)\right)\right)=Z\left(\mathbf{H}_{\bar{A}}\right)$. Due to the bijectivity of $\chi$ we have exactly for those $g$ that $\psi_{x}(g, z g)=\chi(z) \varphi(g)=\varphi(g) \chi(z)=\psi_{x}(g, g z)$ for all $z \in Z\left(Z\left(\mathbf{H}_{\bar{A}}\right)\right)$. This implies that $z g=g z$, i.e. $g \in Z\left(Z\left(Z\left(\mathbf{H}_{\bar{A}}\right)\right)\right)=Z\left(\mathbf{H}_{\bar{A}}\right)$. Hence we get

$$
\varphi^{-1}\left(Z\left(\mathbf{H}_{\bar{A}}\right)\right) \subseteq Z\left(\mathbf{H}_{\bar{A}}\right)
$$

Our assumption $\psi(\mathbf{B}(\bar{A}))=Z\left(\mathbf{H}_{\bar{A}}\right) \times \mathbf{X}_{x \neq m}\left\{e_{\mathbf{G}}\right\}$ implies now $\varphi\left(Z\left(\mathbf{H}_{\bar{A}}\right)\right)=\left\{e_{\mathbf{G}}\right\}$. This again yields $\operatorname{ker} \varphi \subseteq \varphi^{-1}\left(Z\left(\mathbf{H}_{\bar{A}}\right)\right) \subseteq Z\left(\mathbf{H}_{\bar{A}}\right) \subseteq \operatorname{ker} \varphi$, hence

$$
\operatorname{ker} \varphi=Z\left(\mathbf{H}_{\bar{A}}\right) \text {. }
$$

Therefore we have

$$
Z\left(\mathbf{H}_{\bar{A}}\right) \backslash N\left(Z\left(\mathbf{H}_{\bar{A}}\right)\right) \cong \operatorname{im} \varphi \subseteq Z\left(Z\left(\mathbf{H}_{\bar{A}}\right)\right) .
$$

This, however, cannot always be fulfilled. Let, e.g., be $\mathbf{G}=S U(2)$ and $\bar{A}$ be generic. Then $Z\left(\mathbf{H}_{\bar{A}}\right)=Z(S U(2))=\mathbb{Z}_{2}$ and $Z\left(Z\left(\mathbf{H}_{\bar{A}}\right)\right)=N\left(Z\left(\mathbf{H}_{\bar{A}}\right)\right)=S U(2)$. We are looking now for a homomorphism $\varphi: S U(2) \longrightarrow S U(2)$ with $\operatorname{ker} \varphi=\mathbb{Z}_{2}$. By the homomorphism theorem $S O(3) \cong S U(2) / \mathbb{Z}_{2} \cong \operatorname{im} \varphi$ is a subgroup of $S U(2)$. This is a contradiction.[3] Hence, in general, there is no "reasonable" isomorphism of topological groups between $\mathbf{B}(\bar{A}) \backslash N(\mathbf{B}(\bar{A})$ ) and $Z\left(\mathbf{H}_{\bar{A}}\right) \backslash N\left(Z\left(\mathbf{H}_{\bar{A}}\right)\right) \times \boldsymbol{X}_{x \neq m} Z\left(Z\left(\mathbf{H}_{\bar{A}}\right)\right)$.

Finally, we discuss two special cases.

\footnotetext{
${ }^{10}$ In what follows we in general drop the index $m$ in $g_{m}$ and the index $x$ in $z_{x}$.

${ }^{11}$ In general every injective Lie morphism $f: \mathbf{G} \longrightarrow \mathbf{G}$ is already a Lie isomorphism, if $\mathbf{G}$ is a compact Lie group. This one sees as follows: $\operatorname{im} f$ is compact as a continuous image of a compact set, hence closed. Since the image of a homomorphism in general is a subgroup of the target space, im $f$ is Lie subgroup. By the homomorphism theorem [17] we have $\operatorname{im} f \cong \mathbf{G} / \operatorname{ker} f \cong \mathbf{G}$. Hence, $\operatorname{im} f$ as a subgroup of $\mathbf{G}$ has the same dimension and the same number of connected components as $\mathbf{G}$, and thus is equal to $\mathbf{G}$. Therefore $f$ is continuous and bijective, hence a homeomorphism, hence a Lie isomorphism.

${ }^{12}$ Note that $\mathbf{B}(\bar{A})$ due to the special choice of the $h_{\bar{A}}\left(\gamma_{x}\right)$ consists just of the constant $Z\left(\mathbf{H}_{\bar{A}}\right)$-valued gauge transforms.

${ }^{13} S O(3)$ as (an isomorphic image of) a subgroup of $S U(2)$ having the identical dimension and the same number of connected components has to be even equal $S U(2)$. But, this is impossible, because $S O(3)$ and $S U(2)$ are non-isomorphic.
} 
- $\quad \chi(z)=z$ and $\varphi(g)=g$.

The resulting mapping $\psi_{x}(g, z g)=z g$ just corresponds to the restriction $\Psi_{0}$ of the identical map on $\overline{\mathcal{G}}$. This, however, gives a group isomorphism only if $\varphi$ is indeed a map from $N\left(Z\left(\mathbf{H}_{\bar{A}}\right)\right)$ to $Z\left(Z\left(\mathbf{H}_{\bar{A}}\right)\right)$, i.e., these two spaces are equal.

This criterion is fulfilled for instance in the generic stratum. And indeed, in this case $\Psi_{0}$ is a

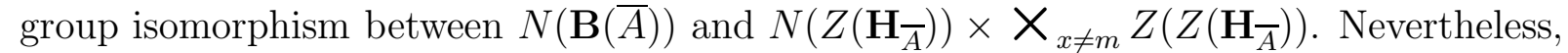
$\Psi_{0}$ factorizes by condition (77) only then to an isomorphism of the quotient groups if $Z(\mathbf{G})=Z\left(\mathbf{H}_{\bar{A}}\right)=\operatorname{ker} \varphi$ equals $\left\{e_{\mathbf{G}}\right\}$.

In the minimal stratum $\Psi_{0}$ is in general no longer an isomorphism because at least for non-abelian $\mathbf{G}$ (i.e., if $\left.\overline{\mathcal{A}}_{\text {gen }} \neq \overline{\mathcal{A}}_{=t_{\min }}\right), N\left(Z\left(\mathbf{H}_{\bar{A}}\right)\right)=\mathbf{G}$ is not equal $Z\left(Z\left(\mathbf{H}_{\bar{A}}\right)\right)=Z(\mathbf{G})$.

- $\quad \chi(z)=z$ and $\varphi(g)=e_{\mathbf{G}}$.

The resulting mapping $\psi_{x}(g, z g)=z$ corresponds here to the homeomorphism $\Psi_{1}$ from Corollary 2.3. In order to turn $\psi$ into a homomorphism, by condition (7), $N\left(Z\left(\mathbf{H}_{\bar{A}}\right)\right)=$ $\operatorname{ker} \varphi=Z\left(\mathbf{H}_{\bar{A}}\right)$ has to hold.

This condition is fulfilled in the minimal stratum. (Then, as can be easily checked, $\Psi_{1}$ is indeed a group isomorphism.)

On the other hand, $\Psi_{1}$ is no isomorphism for generic $\bar{A}$ in the non-abelian case. This is clear, because there we have $N\left(Z\left(\mathbf{H}_{\bar{A}}\right)\right)=\mathbf{G}$, but $Z\left(\mathbf{H}_{\bar{A}}\right)=Z(\mathbf{G})$.

We see again that it is in many cases impossible to find a group isomorphism that additionally does not depend explicitly on the respective stratum containing $\bar{A}$.

\section{B Stratification of $S U(2)^{k}$}

Every $S U(2)$-matrix $A$ can be written uniquely as $A=\left(\begin{array}{cc}a & b \\ -b^{*} & a^{*}\end{array}\right)$ where $a, b \in \mathbb{C}$ are complex numbers fulfilling $|a|^{2}+|b|^{2}=1$. Alternatively such a matrix can be seen as a quaternion $A=a+b \mathrm{j} \in \mathbb{H}$. In this case we also describe $A$ by $a_{0}+a_{1} \mathrm{i}+a_{2} \mathrm{j}+a_{3} \mathrm{k}$ or $a_{0}+\vec{a}$ with $\sum a_{i}^{2}=1$, $a_{i} \in \mathbb{R}$. We have $S U(2) \cong S^{3} \subseteq \mathbb{R}^{4}$.

\section{B.1 Adjoint Action on $S U(2)$}

Let $A, C \in S U(2)$ with $A=a_{0}+a_{1} \mathrm{i}+a_{2} \mathrm{j}+a_{3} \mathrm{k}$ and $C=c_{0}+c_{1} \mathrm{i}+c_{2} \mathrm{j}+c_{3} \mathrm{k}$. It is easy to check that the adjoint action in terms of quaternions is

$$
C^{+} A C=a_{0}+\vec{a}+2\left(\langle\vec{a}, \vec{c}\rangle \vec{c}-\langle\vec{c}, \vec{c}\rangle \vec{a}+c_{0}(\vec{a} \times \vec{c})\right)=a_{0}+\vec{a}+2\left(c_{0}(\vec{a} \times \vec{c})-\vec{c} \times(\vec{a} \times \vec{c})\right) .
$$

Here, $\langle\cdot, \cdot\rangle$ is the canonical scalar product on $\mathbb{R}^{3}$. We determine the stabilizer of $A$.

We have $C^{-1} A C=C^{+} A C=A+2\left(\langle\vec{a}, \vec{c}\rangle \vec{c}-\langle\vec{c}, \vec{c}\rangle \vec{a}+c_{0}(\vec{a} \times \vec{c})\right)$, hence

$$
C \in Z(A) \Longleftrightarrow C^{-1} A C=A \quad \Longleftrightarrow\langle\vec{a}, \vec{c}\rangle \vec{c}-\langle\vec{c}, \vec{c}\rangle \vec{a}+c_{0}(\vec{a} \times \vec{c})=0 .
$$

There are two cases:

1. $\vec{a}=0$, i.e. $A= \pm \mathbf{1}$.

Clearly, the rhs of (9) is true for all $C \in S U(2)$, i.e. $Z(A)=S U(2)$.

2. $\vec{a} \neq 0$, i.e. $A \neq \pm \mathbf{1}$.

Let $C \in Z(A)$. Multiplying the rhs of (9) by $\vec{a}$ we get $\langle\vec{a}, \vec{c}\rangle\langle\vec{c}, \vec{a}\rangle-\langle\vec{c}, \vec{c}\rangle\langle\vec{a}, \vec{a}\rangle=0$. This implies due to $\vec{a} \neq 0$ that $\vec{c}=\mu \vec{a}$ for some $\mu \in \mathbb{R}$. Conversely, every such $C$ is indeed a solution. One easily sees $Z(A) \cong U(1)$. 
In the following we interpret a subset $X$ of the three-dimensional ball $B^{3}$ also as a subset $X:=\left\{A=a_{0}+\vec{a} \in S U(2) \mid \vec{a} \in X\right\}$ of $S U(2)$.

Lemma B.1 For $A=a_{0}+\vec{a} \in S U(2)$ we have $Z(A)=\left\{\begin{array}{ll}B^{3} & \text { for } a_{0}^{2}=1 \\ \mathbb{R} \vec{a} \cap B^{3} & \text { for } a_{0}^{2} \neq 1\end{array}\right.$.

Since every $S U(2)$-matrix can be diagonalized, there is a diagonal matrix in every orbit

Proposition B.2 Every orbit $A \circ S U(2)$ w.r.t. the adjoint action of $S U(2)$ on itself contains a point of the form $a_{0}+\sqrt{1-a_{0}^{2}}$ i. We have $S U(2) / \mathrm{Ad} \cong[-1,1]$ by $[A] \longmapsto \frac{1}{2} \operatorname{tr} A$.

The orbits are the small spheres with constant real part $a_{0}$.

\section{B.2 Adjoint Action on $S U(2)^{2}$}

A crucial point for the investigation of gauge orbit types has been the finiteness lemma for centralizers [13], i.e. every centralizer can be represented as the centralizer of finitely many elements. When we have dealt with the generic stratum we have seen that it is important to generate this way the center of the structure group. How many elements do we need at least for that procedure?

Lemma B.3 We have $Z=\{ \pm \mathbf{1}\} \equiv \mathbb{Z}_{2}$.

Proof $Z=\bigcap_{A \in S U(2)} Z(A)=B^{3} \cap \bigcap_{\vec{a} \in B^{3}} \mathbb{R} \vec{a}=\{\overrightarrow{0}\} \equiv\{ \pm \mathbf{1}\}$ by Lemma B.1. $\quad$ qed

Obviously no single element of $S U(2)$ generates the whole center, but already two elements are sufficient. We only have to guarantee that their centralizers have trivial intersection.

Proposition B.4 There are three orbit types on $S U(2) \times S U(2)$.

Explicitly we have for $A, B \in S U(2)$ :

1. Type $[S U(2)]$

$Z(A, B)=B^{3}$ iff $Z(A)=Z(B)=B^{3}$.

2. Type $[U(1)]$

$Z(A, B)=\mathbb{R} \vec{c} \cap B^{3}$ iff

a) one centralizer equals $B^{3}$ and one equals $\mathbb{R} \vec{c} \cap B^{3}$ or

b) two centralizers equal $\mathbb{R} \vec{c} \cap B^{3}$.

3. Type $\left[\mathbb{Z}_{2}\right]$ (generic elements) $Z(A, B)=\{\overrightarrow{0}\}$ iff $Z(A)=\mathbb{R} \vec{a} \cap B^{3}$ and $Z(B)=\mathbb{R} \vec{b} \cap B^{3}$ where $\vec{a}$ and $\vec{b}$ are non-collinear.

The dimensions of the respective strata are:

- Type $[S U(2)]: 0$;

- Type $[U(1)]: 3$ (case 2.a)] or 4 (case 2.b)]);

- Type $\left[\mathbb{Z}_{2}\right]: 6$.

Clearly, $\left(S U(2)^{2}\right)_{\text {gen }}$ is open and dense in $S U(2)^{2}$. The orbits in the generic stratum are isomorphic to $S U(2) / \mathbb{Z}_{2}$, i.e. are three-dimensional. Hence the quotient space $\left(S U(2)^{2}\right)_{\operatorname{gen}} / \mathrm{Ad}$ is three-dimensional. 
Next, we shall find a continuous section in the generic stratum of $S U(2)^{2}$. What could be a "natural" element describing an orbit $[(A, B)]$ ? Let there be given $(A, B) \in\left(S U(2)^{2}\right)_{\text {gen. }}$ First we are free to use a matrix $C$ for diagonalizing the first component $A$. We get $\left(C^{+} A C, C^{+} B C\right)$. It remains the freedom to act with a second matrix $\Delta_{\beta}$, however, keeping $C^{+} A C$ invariant. Hence, $\Delta_{\beta}$ has to be a diagonal matrix. On the other hand, $\Delta_{\beta}$ has to transform the matrix $C^{+} B C$. Otherwise, $C^{+} B C$ would be a diagonal matrix in contradiction to $Z(A) \cap Z(B)=\{\overrightarrow{0}\}$. Hence, by an appropriate choice of $\Delta_{\beta}$ we can make the secondary diagonal of $\left(C \Delta_{\beta}\right)^{+} B\left(C \Delta_{\beta}\right)$ real. Explicitly we get:

Proposition B.5 In every generic orbit there is a unique element of the form

$$
\left(\left(\begin{array}{cc}
\lambda & 0 \\
0 & \lambda^{*}
\end{array}\right),\left(\begin{array}{cc}
x & \sqrt{1-|x|^{2}} \\
-\sqrt{1-|x|^{2}} & x^{*}
\end{array}\right)\right)
$$

where $|\lambda|=1, \operatorname{Im} \lambda>0$ and $|x|<1$. We call such an element standard form of the orbit (or its elements).

Conversely, every such element defines a unique generic orbit.

Furthermore the mapping $\pi_{F}:\left(S U(2)^{2}\right)_{\text {gen }} \longrightarrow\left(S U(2)^{2}\right)_{\text {gen }}$ assigning to every element its standard form is continuous.

Explicitly, we have for the standard form of $(A, B)=\left(a_{0}+\vec{a}, b_{0}+\vec{b}\right)$ :

$$
\begin{aligned}
& \lambda=a_{0}+\|\vec{a}\| \mathrm{i} \\
& x=b_{0}+\frac{\langle\vec{a}, \vec{b}\rangle}{\|\vec{a}\|} \mathrm{i},
\end{aligned}
$$

where $\langle\cdot, \cdot\rangle$ is again the canonical scalar product on $\mathbb{R}^{3}$.

On the level of quaternions the element above can be written as $\left(\lambda, x+\sqrt{1-|x|^{2}} \mathrm{j}\right)$.

Proof - Existence

Let $A=a_{0}+\vec{a}=a_{0}+a_{1} \mathrm{i}+a_{2} \mathrm{j}+a_{3} \mathrm{k} \neq \pm \mathbf{1}$. Define

$$
\varepsilon:=\frac{a_{1}}{\|\vec{a}\|} \quad \text { and } \quad \delta:=\frac{a_{2}+a_{3} \mathrm{i}}{\sqrt{a_{2}^{2}+a_{3}^{2}}}
$$

as well as

$$
C:=\frac{1}{\sqrt{2}}\left(\begin{array}{cc}
\mathrm{i} \sqrt{1+\varepsilon} & \delta \sqrt{1-\varepsilon} \\
-\delta^{*} \sqrt{1-\varepsilon} & -\mathrm{i} \sqrt{1+\varepsilon}
\end{array}\right) .
$$

One easily checks $C^{+}(A, B) C=\left(\left(\begin{array}{cc}\lambda & 0 \\ 0 & \lambda^{*}\end{array}\right), \widetilde{B}\right)$ with an appropriate matrix $\widetilde{B}$, where $|\lambda|=1$ and $\operatorname{Im} \lambda>0$.⿴囗十丁

Afterwards we choose a $\beta \in \mathbb{C}$ with

$$
\beta^{2}=\frac{\widetilde{b}_{2}+\widetilde{b}_{3} \mathrm{i}}{\sqrt{\tilde{b}_{2}^{2}+\widetilde{b}_{3}^{2}}}
$$

Here let $\widetilde{b}_{i}$ be as usual the quaternionic components of $\widetilde{B}$. Note that the denominator above is always nonzero because $\widetilde{B}$ cannot be a diagonal matrix.

Now, on the one hand, the diagonal matrix $\Delta_{\beta}:=\left(\begin{array}{cc}\beta & 0 \\ 0 & \beta^{*}\end{array}\right)$ commutes with $C^{+} A C$ and, on the other hand, the secondary diagonal in $\Delta_{\beta}^{+} \widetilde{B} \Delta_{\beta}$ is real and positive in the upper right corner, thus, in particular, nonzero. Hence $\left(C \Delta_{\beta}\right)^{+}(A, B)\left(C \Delta_{\beta}\right)$ is of the desired type.

\footnotetext{
${ }^{14} \delta$ can be chosen arbitrarily with norm 1 if $a_{2}=a_{3}=0$.

${ }^{15}$ Note that $\operatorname{Im} \lambda=0$ is impossible because otherwise $\lambda= \pm 1$, i.e. $A= \pm \mathbf{1}$.
} 
Furthermore, one checks that $\lambda$ and $x$ depend indeed in the given manner on $A$ and $B .{ }^{10}$

- Uniqueness

Suppose, there were two such elements $\left(A_{i}, B_{i}\right)=\left(\lambda_{i}, x_{i}+\sqrt{1-\left|x_{i}\right|^{2}} \mathrm{j}\right)$ fulfilling the conditions above. Then there is a $C \in S U(2)$ with $C^{+}\left(A_{1}, B_{1}\right) C=\left(A_{2}, B_{2}\right)$.

- $C^{+} A_{1} C=A_{2}$

Since conjugate quaternions have always the same real part, we have $\operatorname{Re} \lambda_{1}=$ Re $\lambda_{2}$. Hence, $\lambda_{1}=\lambda_{2}$ oder $\lambda_{1}=\lambda_{2}^{*}$. Since $\operatorname{Im~} \lambda_{i}>0$ by assumption, we get $\lambda_{1}=\lambda_{2}$, thus $A_{1}=A_{2}$.

Moreover, that is why $C$ is in $Z\left(A_{1}\right)$ and equals $\mu \in \mathbb{C}$ with $|\mu|=1$.

$-C^{+} B_{1} C=B_{2}$

We have

$$
\begin{aligned}
x_{2}+\sqrt{1-\left|x_{2}\right|^{2}} \mathrm{j} & =B_{2} \\
& =C^{+} B_{1} C \\
& =\mu^{*}\left(x_{1}+\sqrt{1-\left|x_{1}\right|^{2}} \mathrm{j}\right) \mu \\
& =x_{1}+\sqrt{1-\left|x_{1}\right|^{2}} \mu^{*} \mu^{*} \mathrm{j},
\end{aligned}
$$

thus first $x_{1}=x_{2}$. Therefore the expressions containing the roots are equal, and due to $\left|x_{i}\right|<1$ we have $\left(\mu^{*}\right)^{2}=1$, i.e. $\mu= \pm 1$.

Thus, $C= \pm \mathbf{1} \in Z$ and hence $\left(A_{1}, B_{1}\right)=\left(A_{2}, B_{2}\right)$.

- Since we assumed $|x|<1$, every element in the proposition above defines an orbit in the generic stratum.

- The continuity of $\pi_{F}$ is clear because of $\|\vec{a}\| \neq 0$ in the generic stratum.18 qed

We denote the space of all standard forms by $F$. Then $F$ is homeomorphic to the product of the upper open semicircle of $U(1)\left(\lambda\right.$-part) and the upper open hemisphere of $S^{2}$ ( $x$-part), hence is homeomorphic to $\mathbb{R}^{3}$.

Proposition B.6 We have $F \cong\left(S U(2)^{2}\right)_{\text {gen }} /$ Ad.

Proof Let $f: F \longrightarrow\left(S U(2)^{2}\right)_{\text {gen }} /$ Ad be the concatenation of the embedding $\iota$ of $F$ into $\left(S U(2)^{2}\right)_{\text {gen }}$ and the canonical projection $\pi_{2}$ :

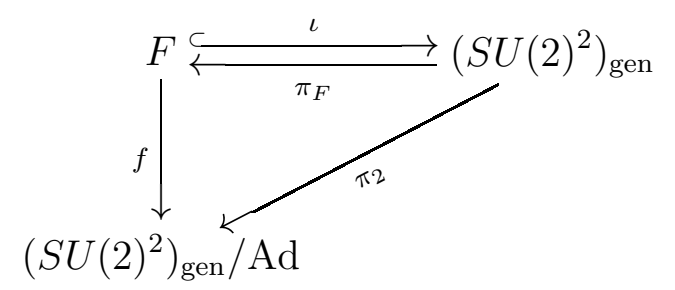

- $f$ is bijective by Proposition B.5.

\footnotetext{
${ }^{16}$ More general, for the action of $C \Delta_{\beta}$ on a matrix $M=m_{0}+\vec{m}$ we have:

$$
\left(C \Delta_{\beta}\right)^{+} M\left(C \Delta_{\beta}\right)=m_{0}+\frac{\langle\vec{a}, \vec{m}\rangle}{\|\vec{a}\|} \mathrm{i}+\frac{\langle\vec{a} \times \vec{b}, \vec{a} \times \vec{m}\rangle}{\|\vec{a} \times \vec{b}\|\|\vec{a}\|} \mathrm{j}+\frac{\langle\vec{a} \times \vec{b}, \vec{m}\rangle}{\|\vec{a} \times \vec{b}\|} \mathrm{k} .
$$

${ }^{17}$ Here, all expressions are quaternionic.

${ }^{18} \mathrm{But}$, note that the map $A \longmapsto C$ itself is not continuous. This can be seen in the special case that $A$ goes to a diagonal matrix, i.e. for $a_{2}, a_{3} \rightarrow 0$ and (here) $a_{1}<0$. Then namely $\varepsilon \rightarrow-1$, i.e., $C$ goes as $\frac{a_{2}+a_{3} \mathrm{i}}{\sqrt{a_{2}^{2}+a_{3}^{2}}} \mathrm{j}$, hence is divergent.
} 
- $f$ is continuous as a concatenation of continuous maps.

- $f^{-1}$ is continuous, because at least locally (around every point) there is a continuous section $s_{2}$ for $\pi_{2}$ such that locally $f^{-1}=\pi_{F} \circ s_{2}$ which implies the local continuity. However, then $f^{-1}$ is globally continuous.

qed

Consequently, the generic stratum of $S U(2)^{2}$ is homeomorphic to $\mathbb{R}^{3} \times S O(3)$ because $S U(2) / \mathbb{Z}_{2} \cong S O(3)$.

We remark that the total orbit space $S U(2)^{2} /$ Ad is homeomorphic to the three-ball $B^{3}$ where the singular strata are simply its boundary $S^{2}$.

\section{B.3 Adjoint Action on $S U(2)^{3}$}

We will show here that the adjoint action on $S U(2)^{3}$ leads to a nontrivial generic stratum. The argument here is again a pure homotopy argument as in the proof of the more general Proposition 9.2. But, here we will explicitly describe the strata on $S U(2)^{3}$ and show that the non-generic strata have codimension 4. (By the way, one easily recognizes that the codimension of the non-generic strata on $S U(2)^{k}$ equals $2(k-1)$.)

Proposition B.7 On $S U(2) \times S U(2) \times S U(2)$ there are three orbit types.

Explicitly we have for $A, B, C \in S U(2)$ :

1. Type $[S U(2)]$

(Dimension 0) $Z(A, B, C)=B^{3}$ iff $Z(A)=Z(B)=Z(C)=B^{3}$.

2. Type $[U(1)]$ $Z(A, B, C)=\mathbb{R} \vec{d} \cap B^{3}$ iff

a) (Dimension 3 ) two centralizers equal $B^{3}$ and one equals $\mathbb{R} \vec{d} \cap B^{3}$ or

b) (Dimension 4) one centralizer equals $B^{3}$ and two equal $\mathbb{R} \vec{d} \cap B^{3}$ or

c) (Dimension 5) three centralizers equal $\mathbb{R} \vec{d} \cap B^{3}$.

3. Type $\left[\mathbb{Z}_{2}\right]$ (generic elements)

$Z(A, B, C)=\{\overrightarrow{0}\}$ iff

a) (Dimension 6) one centralizer equals $B^{3}$ and the remaining two are different and not equal $B^{3}$, or

b) (Dimension 7) two centralizers equal $\mathbb{R} \vec{d} \cap B^{3}$ and one equals $\mathbb{R} \vec{a} \cap B^{3}$, but not all are equal, or

c) (Dimension 9) all centralizers are different and not equal $B^{3}$.

Now we assume that $\left(S U(2)^{3}\right)_{\text {gen }}$ were trivial. Then

hence

$$
\left(S U(2)^{3}\right)_{\text {gen }} \cong\left(S U(2)^{3}\right)_{\text {gen }} / \mathrm{Ad} \times \mathbb{Z}_{2} \backslash S U(2),
$$

$$
\pi_{1}^{\mathrm{h}}\left(\left(S U(2)^{3}\right)_{\text {gen }}\right) \cong \pi_{1}^{\mathrm{h}}\left(\left(S U(2)^{3}\right)_{\text {gen }} / \mathrm{Ad}\right) \times \pi_{1}^{\mathrm{h}}\left(\mathbb{Z}_{2} \backslash S U(2)\right) .
$$

As we have just seen the codimension of $S U(2)^{3} \backslash\left(S U(2)^{3}\right)_{\text {gen }}$ equals 4, i.e., we have $\pi_{1}^{\mathrm{h}}\left(\left(S U(2)^{3}\right)_{\text {gen }}\right)=\pi_{1}^{\mathrm{h}}\left(S U(2)^{3}\right)=\pi_{1}^{\mathrm{h}}(S U(2))^{3}=1$. On the other hand, $\mathbb{Z}_{2} \backslash S U(2)=S O(3)$ and $\pi_{1}^{\mathrm{h}}(S O(3))=\mathbb{Z}_{2}$. Hence,

$$
1 \cong \pi_{1}^{\mathrm{h}}\left(\left(S U(2)^{3}\right)_{\operatorname{gen}} / \mathrm{Ad}\right) \oplus \mathbb{Z}_{2}
$$

which obviously is a contradiction. Hence, $\left(S U(2)^{3}\right)_{\text {gen }}$ is nontrivial. 


\section{Other Kinds of Connections}

In the literatur there are various kinds of regular connections characterized again by the holonomy group $\mathbf{H}_{A}$ :

1. $\mathcal{A}_{\text {irr }}:=\left\{A \in \mathcal{A} \mid \mathbf{H}_{A}=\mathbf{G}\right\}$ (often called irreducible),

2. $\mathcal{A}_{\text {gen }}:=\left\{A \in \mathcal{A} \mid Z\left(\mathbf{H}_{A}\right)=Z(\mathbf{G})\right\}$ (often called generic),

3. $\mathcal{A}_{\text {alm gen }}:=\left\{A \in \mathcal{A} \mid Z\left(\mathbf{H}_{A}\right)\right.$ is discrete $\}$ (we call it almost generic).

Unfortunately, the notations are sometimes diverging. The corresponding sets fulfill $\mathcal{A}_{\text {irr }} \subseteq$ $\mathcal{A}_{\text {gen }} \subseteq \mathcal{A}_{\text {alm gen }} \subseteq \mathcal{A}$ for semi-simple $\mathbf{G}$. If $\mathbf{G}$ is not semi-simple, the center of $\mathbf{G}$ is never discrete [7], hence no centralizer can be discrete. Thus, $\mathcal{A}_{\text {alm gen }}$ would be empty. However, the relation between $\mathcal{A}_{\text {irr }}$ and $\mathcal{A}_{\text {gen }}$ survives for arbitrary $\mathrm{G}$.

As mentioned, e.g., in [0] the inclusions are not always proper. Suppose, e.g., $\mathbf{G}=S U(2)$, then $\mathcal{A}_{\text {irr }}=\mathcal{A}_{\text {alm gen }}$ for simply connected base manifolds $M$ and $\mathcal{A}_{\text {alm gen }}=\mathcal{A}$ for certain $M$ depending on the topology of the bundle $P=P(M, \mathbf{G})$. Moreover, $\mathcal{A}_{\text {gen }}=\mathcal{A}_{\text {alm gen }}$ iff $\mathbf{G}$ is a product of $S U\left(N_{i}\right)$ 's only. However, in the case of generalized connections the relation $\overline{\mathcal{A}}_{\text {irr }} \subset \overline{\mathcal{A}}_{\text {gen }}$ is proper for every (at least one-dimensional, but not necessarily semi-simple) Lie group $\mathbf{G}$. This is a simple consequence the following

Proposition C.1 Let $M$ be at least two-dimensional and $m$ be as always some fixed point in $M$.

For every (abstract) subgroup $H \subseteq \mathbf{G}$ there is a generalized connection whose holonomy group equals $H$.

Proof Let $\bar{A}_{0}$ be the trivial connection, i.e. we have $h_{\bar{A}_{0}}(\gamma)=e_{\mathbf{G}}$ for all $\gamma \in \mathcal{P}$. Furthermore, let $m^{\prime}$ be some point in $M$ different from $m$. We choose a set $E:=\left\{\gamma_{g}\right\}_{g \in H} \subseteq \mathcal{P}$ of edges connecting $m$ and $m^{\prime}$ that do not intersect each other (i.e., the only common points of each two edges are their endpoints $m$ and $m^{\prime}$, respectively). Such an $E$ exists always because $H$ as a subset of $\mathbf{G}$ has at most the cardinality of $\mathbb{R}$. $⿴$ Obviously the set $V_{-}$of all starting points is $\{m\}$, hence finite. By a proposition in 112 there is an $\bar{A} \in \overline{\mathcal{A}}$ with $h_{\bar{A}}\left(\gamma_{g}\right)=g$ for all $g \in H$. In particular, $h_{\bar{A}}\left(\gamma_{g} \gamma_{e_{\mathrm{G}}}^{-1}\right)=g$ for all $g \in H$, hence $H \subseteq \mathbf{H}_{\bar{A}}$. On the other hand, by the definition of $\bar{A}$ (cf. again [12]) and the group property of $H$, we have $h_{\bar{A}}(\gamma) \in H$ for all $\gamma \in \mathcal{P}$. Hence, $\mathbf{H}_{\bar{A}} \subseteq H$. $\quad$ qed

Thus, there are always proper (even countable) subgroups $H \subset \mathbf{G}$ with $Z(H)=Z(\mathbf{G})$ and $H=\mathbf{H}_{\bar{A}}$ for some $\bar{A} \in \overline{\mathcal{A}}$. Up to now, we do not know whether $\overline{\mathcal{A}}_{\text {irr }}$ is open or closed or whatever in $\overline{\mathcal{A}}_{\text {gen }}$. We also do not know whether $\pi: \overline{\mathcal{A}} \longrightarrow \overline{\mathcal{A}} / \overline{\mathcal{G}}$ may be trivial on the irreducible connections. This would be an interesting problem, in particular, because the original paper [27] by Singer on the Gribov ambiguity showed the non-triviality just of the bundle of irreducible (Sobolev) connections.

\section{Slice Theorem implies Bundle Structure}

We are going to prove the following

\footnotetext{
${ }^{19}$ Imagine in some chart (w.l.o.g. equal $\mathbb{R}^{\operatorname{dim} M}$ ) for instance the set $E$ of all field lines between a positive charge in $m$ and a negative charge in $m^{\prime}$. Since there are $\aleph_{1}$ radial directions starting in $m$, there are $\aleph_{1}$ such field lines as well.
} 
Proposition D.1 Let $G$ be a compact topological group and $X$ be a $G$-space.

If a slice theorem holds on $X$, then for every closed subgroup $H \subseteq G$ the stratum $X_{=H}:=\{x \in X \mid \operatorname{Stab}(x) \sim H\}$ is a locally trivial fibre bundle with typical fibre $H \backslash G$ and structure group $H \backslash N(H)$ acting on $H \backslash G$ by left translation.

Recall that a slice theorem holds on $X$ iff for every $x \in X$ there is a "slice" $S \subseteq X$ with $x \in S$ and open saturation $S \circ G$ and there is an equivariant retraction $f$ of $S \circ G$ onto $x \circ G$ with $f^{-1}(\{x\})=S$.

For the proof of the proposition above we need

Lemma D.2 Let $H$ be a closed subgroup of the compact group $G$. Then the group of all equivariant homeomorphisms between $H \backslash G$ and $H \backslash G$ is isomorphic to $H \backslash N(H)$.

Proof For every such $f: H \backslash G \longrightarrow H \backslash G$ there is an $a_{f} \in G$ with $f(H g)=H a_{f}^{-1} g$ for all $g \in G$. [6] Such an $a_{f}$ has to fulfill $a_{f}^{-1} H a_{f} \subseteq H$, hence has to be in $N(H)$. Conversely, every such $a$ determines a unique $f_{a}$ of the type above. By $f(H)=H a_{f}^{-1}$ the map $f \longmapsto a_{f}$ is unique up to multiplication of $a_{f}$ by elements of $H$. Hence $\psi:$ Homeo $^{G}(H \backslash G) \longrightarrow H \backslash N(H), f \longmapsto\left[a_{f}^{-1}\right]_{H}$, is bijective and by $\psi\left(f_{1} \circ f_{2}\right)=$ $\psi\left(f_{1}\right) \psi\left(f_{2}\right)$ even a group isomorphism.

qed

The proof of Proposition D.1 imitates a proof in [6] that was given there in the case of $G$ being a Lie group. Indeed, this restriction is superfluous because one needs only the validity of a slice theorem.

\section{Proof Proposition D.1}

- Since all strata are invariant under $G$, the validity of a slice theorem on $X$ implies immediately that on the stratum $X_{=[H]}$ considered as a $G$-space. Hence, we can assume that all points in $X$ have type $[H]$.

- $X$ is a locally trivial fibre bundle.

We construct around every $x \in X$ with $\operatorname{Stab}(x)=H$ a bundle chart. (Obviously there is such an $x$ in every orbit.) For this, let $S$ be a slice through $x$ and $f: S \circ G \longrightarrow x \circ G$ be the corresponding equivariant retraction, and let $s \in S$ and $h \in G_{s}:=\{h \in G \mid s \circ h=s\} . x=f(s)=f(s \circ h)=f(s) \circ h=x \circ h$ implies $h \in H$, hence $G_{s} \subseteq H$. By assumption all stabilizers on $X$ are conjugate, we have $G_{s}=H$. Thus, $H$ acts trivially on $S$, hence, $S \circ G \cong S \times_{H} G \cong S \times H \backslash G$ as $G$-spaces.

Moreover, $S \cong S / H \cong(S \circ G) / G$ : Let $c: S \longrightarrow(S \circ G) / G$ with $c(s)=[s]$.

$-\quad c$ is surjective by construction.

- $c$ is injective, since $c\left(s_{1}\right)=c\left(s_{2}\right)$ implies $s_{1} \circ g=s_{2}$ for some $g \in G$, hence (using $f$ ) $x \circ g=x$, thus $g \in H=G_{s}$ and finally $s_{2}=s_{1} \circ g=s_{1}$.

$-c$ is continuous.

- $c$ is closed, because first for every closed $U \subseteq S$ (by the closedness of $f^{-1}(\{x\})=S$ in $\left.S \circ G\right) U$ in $S \circ G$, hence (by the compactness of $G$ ) also $U \circ G$ in $S \circ G$ is closed, and second $\pi: S \circ G \longrightarrow(S \circ G) / G$ is closed.

- $c$ is open, because it is closed and bijective. 
Using that identification we get a chart map by the following commutative diagram:

$$
\chi_{S}:
$$

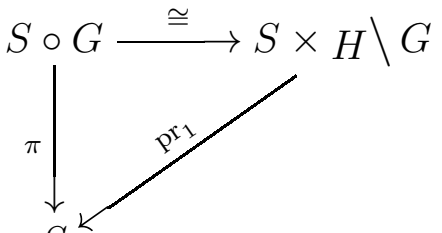

- $H \backslash N(H)$ is the structure group of $\underset{X}{X}$.

$$
S^{K}
$$

Let $\chi_{S}$ and $\chi_{T}$ be two chart mappings as above. We define for all $x \in S \cap T$ using

an equivariant homeomorphism

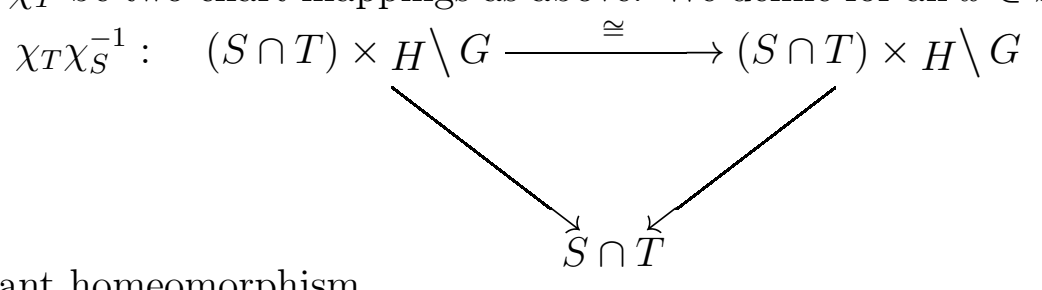

$$
\vartheta_{x}: H \backslash G \longrightarrow H \backslash G \text { by } \chi_{T} \chi_{S}^{-1}(x, H g)=\left(x, \vartheta_{x}(H g)\right) \text {. }
$$

According to the proof of Lemma D.2, $\vartheta_{x}$ corresponds via $\vartheta_{x}(H)=H a_{\vartheta_{x}}^{-1}$ to a unique $\rho_{T}^{S}(x):=\left[a_{\vartheta_{x}}\right]^{-1} \in H \backslash N(H)$. Hence $\chi_{S}^{-1}(x, H g)=\chi_{T}^{-1}\left(x, \rho_{T}^{S}(x) \circ H g\right)$.

We are now left with the proof of the continuity of $\rho_{T}^{S}: S \cap T \longrightarrow H \backslash N(H)$. But, this we get easily from the continuity of $\chi_{T} \chi_{S}^{-1}$ by means of

$$
\begin{array}{ccccccc}
S \cap T & \stackrel{\iota}{\longleftrightarrow} & (S \cap T) \times H \backslash H & \stackrel{\chi_{T} \chi_{S}^{-1}}{\longrightarrow} & (S \cap T) \times H \backslash N(H) & \stackrel{\mathrm{pr}_{2}}{\longrightarrow} & H \backslash N(H) . \\
x & \longmapsto & (x, H) & \longmapsto & \left(x, H a_{\vartheta_{x}}^{-1}\right) & \longmapsto & \rho_{T}^{S}(x) \\
& & & & & &
\end{array}
$$

\section{E Codimension of Nongeneric Strata}

Let $\mathbf{G}$ be a compact Lie group acting smoothly on a manifold $M$. By $\left(x_{1}, \ldots, x_{k}\right) \circ g:=$ $\left(x_{1} \circ g, \ldots, x_{k} \circ g\right)$ for every $k \in \mathbb{N}_{+}$we define an (again smooth) action of $\mathbf{G}$ on $M^{k}$. Obviously, the types of this action are again conjugacy classes of closed subgroups of $\mathbf{G}$. Now we have

Proposition E.1 Let $\mathbf{G}$ and $M$ be as above, $\mathbf{H}$ be a closed subgroup of $\mathbf{G}$ and $k \in \mathbb{N}_{+}$be arbitrary. Then we have

$$
\max _{[\mathbf{K}]<[\mathbf{H}]} \operatorname{dim}\left(M^{k}\right)_{\mathbf{K}} \leq k \max _{[\mathbf{K}]<[\mathbf{H}]} \operatorname{dim} M_{\mathbf{K}}
$$

Proof Let $\vec{x}:=\left(x_{1}, \ldots, x_{k}\right) \in\left(M^{k}\right)_{\mathbf{K}}$ for some $[\mathbf{K}]<[\mathbf{H}]$. For some appropriate $g \in \mathbf{G}$ we have $g^{-1} \mathbf{K} g=\operatorname{Stab}(\vec{x})=\bigcap_{i} \operatorname{Stab}\left(x_{i}\right) \subseteq \operatorname{Stab}\left(x_{i}\right)$ for all $i$. Thus, $\left[\operatorname{Stab}\left(x_{i}\right)\right] \leq[\mathbf{K}]<$ $[\mathbf{H}]$, hence $x_{i} \in \bigcup_{\left[\mathbf{K}_{i}\right] \leq[\mathbf{K}]} M_{\mathbf{K}_{i}}$.

Consequently,

$$
\left(M^{k}\right)_{\mathbf{K}} \subseteq \underset{i}{X}\left(\bigcup_{\left[\mathbf{K}_{i}\right] \leq[\mathbf{K}]} M_{\mathbf{K}_{i}}\right)=\bigcup_{\left[\mathbf{K}_{i}\right] \leq[\mathbf{K}]} M_{\mathbf{K}_{1}} \times \cdots \times M_{\mathbf{K}_{k}} .
$$

Since there are only finitely many orbit types on $M^{k}$ [26], we get 


$$
\begin{aligned}
\operatorname{dim}\left(M^{k}\right)_{\mathbf{K}} & \leq \max _{\left[\mathbf{K}_{i}\right] \leq[\mathbf{K}]} \operatorname{dim} M_{\mathbf{K}_{1}} \times \cdots \times M_{\mathbf{K}_{k}} \\
& =k \max _{\left[\mathbf{K}^{\prime}\right] \leq[\mathbf{K}]} \operatorname{dim} M_{\mathbf{K}^{\prime}} \\
& \leq k \max _{\left[\mathbf{K}^{\prime}\right]<[\mathbf{H}]} \operatorname{dim} M_{\mathbf{K}^{\prime}} .
\end{aligned}
$$

In particular, we have

$$
\max _{[\mathbf{K}]<[\mathbf{H}]} \operatorname{dim}\left(M^{k}\right)_{\mathbf{K}} \leq k \max _{[\mathbf{K}]<[\mathbf{H}]} \operatorname{dim} M_{\mathbf{K}}
$$

Corollary E.2 We have

$$
\min _{[\mathbf{K}]<[\mathbf{H}]} \operatorname{codim}_{M^{k}}\left(M^{k}\right)_{\mathbf{K}} \geq k \min _{[\mathbf{K}]<[\mathbf{H}]} \operatorname{codim}_{M} M_{\mathbf{K}}
$$

\section{F Proof of Proposition 7.3}

First we discuss properties of so-called fundamental systems of connected hyphs, i.e., certain free generating systems for the group of based paths spanned by that hyph. For that purpose, we consider hyphs as abstract graphs. This makes graph-theoretical concepts applicable, like the notion of maximal trees.

Definition F.1 • Let $v=\left\{e_{1}, \ldots, e_{Y}\right\}$ be a connected hyph with $m \in \mathbf{V}(v)$ and $v^{\prime}$ be a maximal tree in $v$. Moreover, let $\phi:[1, n] \longrightarrow[1, Y]$ be an injective function, such that $v^{\prime}=v \backslash\left\{e_{\phi(1)}, \ldots, e_{\phi(n)}\right\}$. (This intricate definition is necessary, because $v^{\prime}$ is to be a hyph again and the hyph property requires a certain ordering of the edges of a hyph.)

$\boldsymbol{\alpha} \subseteq \mathcal{H G}$ is called weak $v^{\prime}$-fundamental system for $v$ iff for every $i=1, \ldots, n$ the path $\alpha_{i}$ can be expressed as a product of three paths, whereas the first and the third path are contained in $\mathcal{P}_{\left\{e_{\phi(1)}, \ldots, e_{\phi(i-1)}\right\} \cup v^{\prime}}$ and the second path equals the edge $e_{\phi(i)}$.

The edge $e_{\phi(i)}$ is called free edge of $\alpha_{i}$.

- $\boldsymbol{\alpha} \subseteq \mathcal{H G}$ is called weak fundamental system iff there are a connected hyph $v$ and a maximal tree $v^{\prime}$ in $v$, such that $\boldsymbol{\alpha}$ is a weak $v^{\prime}$-fundamental system for $v$.

Here, $\mathcal{P}_{\boldsymbol{\gamma}}$ denotes the subgroupoid of $\mathcal{P}$ generated by $\boldsymbol{\gamma} \subseteq \mathcal{P}$. Analogously, $\mathcal{H G}_{\boldsymbol{\beta}}$ is defined. We have obviously

Lemma F.1 Every connected hyph possesses a weak fundamental system.

The basic properties of weak fundamental systems are summarized in

Proposition F.2 Let $v$ be a connected hyph with $m \in \mathbf{V}(v)$, and let $\boldsymbol{\alpha}$ be a weak fundamental system of $v$. Then the following holds:

1. $\mathcal{P}_{\boldsymbol{\alpha}}$ is freely generated by $\boldsymbol{\alpha}$ and equals $\mathcal{H} \mathcal{G}_{v}$;

2. $\pi_{\boldsymbol{\alpha} *} \mu_{0}=\mu_{\text {Haar }}^{\# \boldsymbol{\alpha}}$

3. $\pi_{\boldsymbol{\alpha}}: \overline{\mathcal{A}} \longrightarrow \mathrm{G}^{\# \boldsymbol{\alpha}}$ is surjective.

Consequently, every weak fundamental system of a connected hyph is a weak hyph. 
Proof By assumption there is a maximal tree $v^{\prime}$ in $v=\left\{e_{1}, \ldots, e_{Y}\right\}$, such that $\boldsymbol{\alpha}$ is a $v^{\prime}$ fundamental system of $v$. In order to avoid more complicated expressions, we simply assume $v^{\prime}=\left\{e_{n+1}, \ldots, e_{Y}\right\}$.

1. Obviously $\mathcal{P}_{\boldsymbol{\alpha}} \subseteq \mathcal{H} \mathcal{G}_{v}$.

- We show $\mathcal{H} \mathcal{G}_{v} \subseteq \mathcal{P}_{\boldsymbol{\alpha}}$ inductively w.r.t. $n=\# \boldsymbol{\alpha}=\# v-\# v^{\prime}$.

For $n=0$ the statement is trivial. Now, let $n>0$ and $\gamma \in \mathcal{H G}_{v}$, i.e. $\gamma=$ $\gamma_{1} e_{n}^{\eta_{1}} \gamma_{2} e_{n}^{\eta_{2}} \cdots e_{n}^{\eta_{q}} \gamma_{q+1}$ with $\gamma_{j} \in \mathcal{P}_{\widetilde{v}}$ and $\eta_{j}= \pm 1$ for all $j$. Here $\widetilde{v}:=v \backslash\left\{e_{n}\right\}$. Let $\delta_{+1}$ and $\delta_{-1}$ be those paths in $\mathcal{P}_{\widetilde{v}}$ that fulfill $\alpha_{n}=\delta_{-1} e_{n} \delta_{+1}$. Hence,

$$
\gamma=\underbrace{\gamma_{1} \delta_{-\eta_{1}}^{-\eta_{1}}}_{\in \mathcal{H} \mathcal{G}_{\widetilde{v}}} \alpha_{n}^{\eta_{1}} \underbrace{\delta_{\eta_{1}}^{-\eta_{1}} \gamma_{2} \delta_{-\eta_{2}}^{-\eta_{2}}}_{\in \mathcal{H} \mathcal{G}_{\widetilde{v}}} \alpha_{n}^{\eta_{2}} \delta_{\eta_{2}}^{-\eta_{2}} \cdots \delta_{-\eta_{q}}^{-\eta_{q}} \alpha_{n}^{\eta_{q}} \underbrace{\delta_{\eta_{q}}^{-\eta_{q}} \gamma_{q+1}}_{\in \mathcal{H} \mathcal{G}_{\widetilde{v}}} .
$$

By $e_{n} \notin v^{\prime}$, also $v^{\prime}$ is a maximal tree for $\widetilde{v}$ and, in particular, $\widetilde{v}$ is connected. Now, $\boldsymbol{\alpha} \backslash\left\{\alpha_{n}\right\}$ is a weak $v^{\prime}$-fundamental system for $\widetilde{v}$. Due to $\# \widetilde{v}-\# v^{\prime}=$ $\# v-\# v^{\prime}-1=n-1$ we have by the induction hypothesis $\mathcal{H G}_{\widetilde{v}}=\mathcal{P}_{\boldsymbol{\alpha} \backslash\left\{\alpha_{n}\right\}}$. Equation (10) implies $\gamma \in \mathcal{P}_{\boldsymbol{\alpha}}$.

- Consequently, $\boldsymbol{\alpha}$ is a generating system of the free group $\mathcal{H G}_{v}$, whose rank equals $\# v-\# v^{\prime}$, hence $\# \boldsymbol{\alpha}$. Thus, $\boldsymbol{\alpha}$ is free. 22]

2. We have

$$
\begin{aligned}
& \pi_{\boldsymbol{\alpha}}^{v}: \quad \mathbf{G}^{Y} \quad \longrightarrow \quad \mathbf{G}^{n}, \\
& \left(g_{1}, \ldots, g_{Y}\right) \longmapsto\left(\cdots g_{1} \cdots, \ldots, \cdots g_{n} \cdots\right)
\end{aligned}
$$

where the $\cdots$ in $\cdots g_{i} \cdots$ denote a product of some $g_{j}$ or $g_{j}^{-1}$ with $j>n$ or $j<i$. Now, we get for all $f \in C\left(\mathbf{G}^{n}\right)$ using successively the structure of $\cdots g_{i} \cdots$ and the translation invariance and the normalization of the Haar measure

$$
\begin{aligned}
& \int_{\mathbf{G}^{Y}}\left(\pi_{\boldsymbol{\alpha}}^{v}\right)^{*} f \mathrm{~d} \mu_{\text {Haar }}^{Y} \\
= & \int_{\mathbf{G}^{Y}} f\left(\pi_{\boldsymbol{\alpha}}^{v}\left(g_{1}, \ldots, g_{Y}\right)\right) \mathrm{d} \mu_{\text {Haar }, 1} \cdots \mathrm{d} \mu_{\text {Haar }, Y} \\
= & \int_{\mathbf{G}^{Y}} f\left(\cdots g_{1} \cdots, \ldots, \cdots g_{n-1} \cdots, \cdots g_{n} \cdots\right) \mathrm{d} \mu_{\text {Haar }, 1} \cdots \mathrm{d} \mu_{\text {Haar }, Y} \\
= & \int_{\mathbf{G}^{Y}} f\left(\cdots g_{1} \cdots, \ldots, \cdots g_{n-1} \cdots, g_{n}\right) \mathrm{d} \mu_{\text {Haar }, 1} \cdots \mathrm{d} \mu_{\text {Haar }, Y} \\
\vdots & \\
= & \int_{\mathbf{G}^{n}} f\left(g_{1}, \ldots, g_{n}\right) \mathrm{d} \mu_{\text {Haar }, 1} \cdots \mathrm{d} \mu_{\text {Haar }, n} \\
= & \int_{\mathbf{G}^{n}} f \mathrm{~d} \mu_{\text {Haar }}^{n} .
\end{aligned}
$$

Since $\pi_{v}$ projects the induced Haar measure onto the Haar measure [12], we have $\pi_{\boldsymbol{\alpha} *} \mu_{0}=\left(\pi_{\boldsymbol{\alpha}}^{v}\right)_{*}\left(\pi_{v *} \mu_{0}\right)=\left(\pi_{\boldsymbol{\alpha}}^{v}\right)_{*} \mu_{\text {Haar }}^{Y}=\mu_{\text {Haar }}^{n}$.

3. Let $\left(g_{1}, \ldots, g_{n}\right) \in \mathbf{G}^{n}$. By assumption we can express every $e_{i}, i \leq n$, as a product of $\alpha_{i}$ with appropriate paths in $\mathcal{P}_{\left\{e_{1}, \ldots, e_{i-1}\right\} \cup v^{\prime}}$. Now, we choose $\delta_{i, \pm} \in \mathcal{P}_{v^{\prime}}$, such that $\delta_{i,-} e_{i} \delta_{i,+}$ is a path in $\mathcal{H} \mathcal{G}$. But, then $\delta_{i,-} e_{i} \delta_{i,+}$ equals a product of a closed path in $\mathcal{P}_{\left\{e_{1}, \ldots, e_{i-1}\right\} \cup v^{\prime}}$, the path $\alpha_{i}$ and a further closed path $\mathcal{P}_{\left\{e_{1}, \ldots, e_{i-1}\right\} \cup v^{\prime}}$. By the first step $\mathcal{H}_{\left\{e_{1}, \ldots, e_{i-1}\right\} \cup v^{\prime}}=\mathcal{P}_{\left\{\alpha_{1}, \ldots, \alpha_{i-1}\right\}}$. Hence, $\delta_{i,-} e_{i} \delta_{i,+}=\prod_{k_{i}}^{K_{i}} \alpha_{k\left(k_{i}, i\right)}^{ \pm 1} \alpha_{i} \prod_{l_{i}}^{L_{i}} \alpha_{l\left(l_{i}, i\right)}^{ \pm 1}$ for some functions $k$ and $l$, that fulfill always $k\left(k_{i}, i\right)<i$ and $l\left(l_{i}, i\right)<i$. 
Since $\pi_{v}$ is surjective for every hyph [12], there is an $\bar{A} \in \overline{\mathcal{A}}$, such that $h_{\bar{A}}\left(e_{i}\right)=e_{\mathbf{G}}$ for all $i>n$ and $h_{\bar{A}}\left(e_{i}\right)=h_{\bar{A}}\left(\delta_{i,-}\right)^{-1}\left(\prod_{k_{i}}^{K_{i}} g_{k\left(k_{i}, i\right)}^{ \pm 1}\right) g_{i}\left(\prod_{l_{i}}^{L_{i}} g_{l\left(l_{i}, i\right)}^{ \pm 1}\right) h_{\bar{A}}\left(\delta_{i_{i,+}}\right)^{-1}$ for all $i \leq n$ (defined inductively, since $\delta_{i, \pm}$ may run through $e_{i^{\prime}}$ with $i^{\prime}<i$ or $i^{\prime}>n$ ). By construction we have $h_{\bar{A}}\left(\alpha_{i}\right)=g_{i}$ for all $i=1, \ldots, n$.

qed

Finally, we have

\section{Proof Proposition 7.3}

First we choose a $\boldsymbol{\beta} \subseteq \mathcal{H} \mathcal{G}$ with $Z\left(h_{\bar{A}}(\boldsymbol{\beta})\right)=Z\left(\mathbf{H}_{\bar{A}}\right)$. Since $\boldsymbol{\beta}$ is obviously connected, there is a connected hyph $v$, such that every path in $\boldsymbol{\beta}$ can be expressed by paths in $v$. (The existence of a hyph is proven in [12], the connectedness is a simple consequence [14.) Lemma F.1 guarantees the existence of a weak fundamental system $\boldsymbol{\alpha} \subseteq \mathcal{H G}$ for $v$. By Proposition F.2, $\boldsymbol{\alpha}$ is a weak hyph. Since every $\beta \in \mathcal{H G}$ is a closed path in $v$ by construction, it can be written as a product of paths in $\boldsymbol{\alpha}$ (and their inverses). Hence, $h_{\bar{A}}(\beta)$ is a product of $h_{\bar{A}}\left(\alpha_{i}\right)$ and their inverses.

Now, let $g \in Z\left(h_{\bar{A}}(\boldsymbol{\alpha})\right)$. Then $g$ commutes with all finite products of $h_{\bar{A}}\left(\alpha_{i}\right)$ and their inverses as well; thus, in particular, $g \in Z\left(h_{\bar{A}}(\beta)\right)$. This implies $Z\left(\mathbf{H}_{\bar{A}}\right) \subseteq$ $Z\left(h_{\bar{A}}(\boldsymbol{\alpha})\right) \subseteq \bigcap_{\beta \in \boldsymbol{\beta}} Z\left(h_{\bar{A}}(\beta)\right)=Z\left(h_{\bar{A}}(\boldsymbol{\beta})\right)=Z\left(\mathbf{H}_{\bar{A}}\right)$, hence $Z\left(h_{\bar{A}}(\boldsymbol{\alpha})\right)=Z\left(\mathbf{H}_{\bar{A}}\right)$.

qed

\section{G Homeomorphy Criterion}

Proposition G.1 Let $X$ be a topological space, $Y$ be a Hausdorff space and $f: X \longrightarrow Y$ be a bijective and continuous mapping.

If every point in $Y$ has a compact neighbourhood whose preimage is compact again, then $f$ is a homeomorphism.

Proof Let $x \in X$. By assumption there is a compact neighbourhood $W \subseteq Y$ of $f(x)$ such that $V:=f^{-1}(W)$ is compact again. By continuity of $f, V$ is a neighbourhood of $x$. Hence, $\left.f\right|_{V}: V \longrightarrow W$ is a bijective and continuous mapping of a compact space into a Hausdorff space, hence a homeomorphism.

Now we show that $f^{-1}$ is continuous in $f(x)$. For this, let $U$ be an open neighbourhood of $x$ in $X$ and $W_{0} \subseteq W$ be an open neighbourhood of $f(x)$ in $Y$. If one sets $V_{0}:=f^{-1}\left(W_{0}\right)$, then $U \cap V_{0}$ is an open neighbourhood of $x$ in $X$, hence in $V$ as well. Consequently, $\left.f\right|_{V}\left(U \cap V_{0}\right)=f\left(U \cap V_{0}\right)$ is open w.r.t. $f(V)=W$. By $f\left(V_{0}\right)=W_{0}$ and the bijectivity of $f$ we have $f(U) \cap W_{0}=f(U) \cap f\left(V_{0}\right)=f\left(U \cap V_{0}\right) \subseteq W_{0}$. But, $f(U) \cap W_{0}$ is open in $W$, hence in $W_{0}$ as well and thus (by the openness of $W_{0}$ in $Y$ ) in $Y$, too. Hence, $f(U) \supseteq f(U) \cap W_{0} \ni f(x)$ is a neighbourhood of $f(x)$ in $Y$. Since this is true for all open neighbourhoods $U$ of $x, f^{-1}$ is continuous.

qed

\section{References}

[1] A. Ashtekar and C. J. Isham, Class. Quant. Grav. 9 (1992) 1433-1468.

[2] A. Ashtekar and J. Lewandowski, In: Knots and Quantum Gravity (ed. by J. C. Baez, Oxford University Press, Oxford, 1994), e-print: gr-qc/9311010. 
[3] A. Ashtekar and J. Lewandowski, J. Math. Phys. 36 (1995) 2170-2191.

[4] J. C. Baez and S. Sawin, J. Funct. Anal. 150 (1997) 1-26.

[5] M. Baum and Th. Friedrich, Ann. Glob. Anal. Geom. 3 (1985) 120-128.

[6] G. E. Bredon, Introduction to Compact Transformation Groups (Academic Press, Inc., New York, 1972).

[7] Th. Bröcker and T. tom Dieck, Representations of Compact Lie Groups (Springer, New York, 1985).

[8] M. Daniel and C. M. Viallet, Phys. Lett. 76B (1978) 458-460.

[9] J. Dieudonné, Grundzüge der modernen Analysis, Bd. 3 (VEB Deutscher Verlag der Wissenschaften, 1976).

[10] J. Dieudonné, Grundzüge der modernen Analysis, Bd. 4 (VEB Deutscher Verlag der Wissenschaften, 1976).

[11] L. D. Faddeev and V. N. Popov, Phys. Lett. B25 (1967) 29-30.

[12] Ch. Fleischhack, MIS-Preprint 3/2000, math-ph/0001007, to appear in J. Geom. Phys.

[13] Ch. Fleischhack, Commun. Math. Phys. 214 (2000) 607-649.

[14] Ch. Fleischhack, Mathematische und physikalische Aspekte verallgemeinerter Eichfeldtheorien im Ashtekarprogramm (Dissertation, Universität Leipzig, 2001).

[15] V. N. Gribov, Nucl. Phys. B139 (1978) 1-19.

[16] S. Helgason, Differential Geometry, Lie Groups, and Symmetric Spaces (Academic Press, Inc., San Diego, 1978).

[17] J. Hilgert und K.-H. Neeb, Lie-Gruppen und Lie-Algebren (Friedr. Vieweg \& Sohn, Braunschweig/Wiesbaden, 1991).

[18] D. Husemoller, Fibre Bundles (Springer-Verlag, New York, 1994).

[19] J. L. Kelley, General Topology (D. van Nostrand Company, Inc., Toronto, New York, London, 1955).

[20] W. Kondracki and J. Rogulski, Dissertationes mathematicae 250 (1985).

[21] W. Kondracki and P. Sadowski, J. Geom. Phys. 3 (1986) 421-434.

[22] A. G. Kurosch, Gruppentheorie 1 (Akademie-Verlag, Berlin, 1970).

[23] P. K. Mitter, Geometry of the space of gauge orbits and the Yang-Mills dynamical system. Lectures given at Cargèse Summer Inst. on Recent Developments in Gauge Theories, Cargèse, France, Aug 26-Sep 8, 1979. 
[24] Дж. Милнор и Дж. Сташеф, Характеристические Классы (Издательство «Мир», Москва, 1979).

[25] P. K. Mitter and C. M. Viallet, Commun. Math. Phys. 79 (1981) 457-472.

[26] A. L. Onishchik (ed.), Lie Groups and Lie Algebras I (Encyclopaedia of Mathematical Sciences 20) (Springer-Verlag, Berlin, 1993).

[27] I. M. Singer, Commun. Math. Phys. 60 (1978) 7-12.

[28] J. H. C. Whitehead, Annals of Math. 41 (1940) 809-824.

[29] H. Whitney, Geometric Integration Theory (Princeton University Press, Princeton, New Jersey, 1957). 